\title{
Cooperation or Confrontation? -- Prediction of COVID-19 Pandemic Situation via Deep Learning
}

\section{Zhonghua Hong}

Shanghai Ocean University

\section{Ziyang Fan}

Shanghai Ocean University

Xiaohua Tong ( $\nabla$ xhtong@tongji.edu.cn )

Tongji University

\section{Ruyan Zhou}

Shanghai Ocean University

\section{Yun Zhang}

Shanghai Ocean University

\section{Yanling Han}

Shanghai Ocean University

\section{Jing Wang}

Shanghai Ocean University

\section{Shuhu Yang}

Shanghai Ocean University

Lijun Xu

Shanghai Ocean University

Kuifeng Luan

Shanghai Ocean University

Huan Xie

Tongji University

\section{Yongjiu Feng}

Tongji University

\section{Shijie Liu}

Tongji University

\section{Peng Chen}

Tongji University

Yanmin Jin

Tongji University

\section{Xiong $\mathrm{Xu}$}

Tongji University

Sicong Liu 
Tongji University

Chao Wei

Tongji University

Chao Wang

Tongji University

\section{Research Article}

Keywords: Confrontation, Pandemic Situation, Second World War, forecasts

Posted Date: December 28th, 2020

DOl: https://doi.org/10.21203/rs.3.rs-128767/v1

License: (c) (7) This work is licensed under a Creative Commons Attribution 4.0 International License. Read Full License 


\title{
Cooperation or confrontation? Prediction of COVID-19 pandemic situation via deep learning
}

\author{
Zhonghua Hong ${ }^{1,2}$, Ziyang Fan ${ }^{1}$, Xiaohua Tong, ${ }^{2, *}$, Ruyan Zhou ${ }^{1}$, Yun Zhang ${ }^{1}$, Yanling Han ${ }^{1}$, Jing Wang ${ }^{1}$, \\ Shuhu Yang ${ }^{1}$, Lijun Xu${ }^{1}$, Kuifeng Luan ${ }^{1}$, Huan Xie ${ }^{2}$, Yongjiu Feng ${ }^{2}$, Shijie Liu ${ }^{2}$, Peng Chen ${ }^{2}$, Yanmin Jin ${ }^{2}$, \\ Xiong $\mathrm{Xu}^{2}$, Sicong $\mathrm{Liu}^{2}$, Chao Wei ${ }^{2}$, Chao Wang ${ }^{2}$
}

${ }^{1}$ College of Information Technology, Shanghai Ocean University, 999 Huchenghuan Road, Shanghai, China

${ }^{2}$ College of Surveying and Geo-Informatics, Tongji University, 1239 Siping Road, Shanghai, China

*Correspondence to Xiaohua Tong [xhtong@tongji.edu.cn]

\begin{abstract}
The COVID-19 pandemic is the most serious catastrophe since the Second World War. To more accurately observe the epidemic under the influence of policies and provide policy adjustments before the official presidential transition in the United States, we use a three-layer superimposed Long-Short-Term-Memory (LSTM) model to predict the epidemic development trend to mid-January, 2021. The proposed model provides more accuracy and stability relative to Susceptible-ExposedInfective-Recovered (SEIR), modified stacked au-to-encoder, and single-layer LSTM models. The performance effects of the measures in China and five countries with severe epidemics are analysed and summarised. The model shows that the error rate of China, five countries and the world is less than $1.4 \%$. According to forecasts, the epidemic situations in the United States, India, and Brazil, caused by untimely, inappropriate policies, lax regulations and insufficient public cooperation, remain very severe, with cases continuing to increase by tens of thousands. The number of cumulative confirmed cases worldwide will exceed 84.58 million by mid-January, 2021; however, the mortality rate will gradually decrease. Based on analysis of measures (including China's effective prevention and control policies), we found that there are performed tremendous different efficiency even using same positive policy for different countries because of various cooperation between people and governments. It is essential to maintain self-protection to prevent the epidemic from deterioration or regenerating, especially, wearing mask and maintaining a safe distance.
\end{abstract}

\section{Introduction}

The coronavirus disease 2019 (COVID-19) is brought on by infection from Severe Acute Respiratory Syndrome (SARS) Coronavirus 2, and the reports of related cases were first released by Wuhan, Hubei Province, China in December 2019 ${ }^{1}$. The widespread COVID-19 epidemic is a serious threat, and has become one of the most challenging global catastrophes facing mankind since the Second World War ${ }^{2}$. On March 11, 2020, the global development of COVID-19 was assessed by the World Health Organization (WHO) as having met the characteristics of a pandemic ${ }^{2}$. The mortality rate of COVID-19 is estimated to be between 2\% and 5\%, i.e. lower than that of SARS and Middle East Respirator Syndrome ${ }^{4,5}$. However, COVID-19 has a higher infection rate than bat-like SARS, and its pathogenicity is between that of SARS and bat-like SARS $^{6}$. Patients with COVID-19 generally have symptoms such as fever, dry cough, fatigue and dyspnea, the immunological function is weakened, and in severe cases, respiratory failure and death may occur $^{7-9}$. The heart may be damaged and develop myocardial inflammation after recovery from COVID-199. In addition, the slowdown or stagnation of national economies during the epidemic prevention period has caused a deceleration in global economic growth ${ }^{11}$, along with aggravating unemployment and hunger ${ }^{12}$. Some national leaders have been infected with the virus, thereby affecting the implementation of policies and management. 
On January 15, 2020, the Chinese Center for Disease Control and Prevention (China CDC) initiated a first-level emergency response ${ }^{13}$. China began implementing measures since January 23, 2020 ${ }^{14}$, and the epidemic had stabilized by March, 2020. In contrast, the current epidemic situation overseas is not very optimistic. The number of infected people continues to maintain substantial increase of tens of thousands. According to the report by the WHO on December 6, more than 200 countries, territory and regions have been invaded by COVID-19, the global epidemic has infected more than 65.8 million people, with 1523656 deaths, and the epidemic in the Europe, Americas and South-East Asia are the worst, especially in the United States and India ${ }^{15}$.

At present, 52 candidate vaccines have entered phase clinical trials; 13 of these have entered the third phase ${ }^{16}$. It is expected that vaccines may be applied by the end of 2020. In addition, countries with outbreaks have taken many measures, such as city lockdowns, banning unimportant gatherings, and business opening in advance, but the effect of the policies are unclear $^{17}$. The president of the United States will officially transition on January 20, 2021, and new policies affecting the epidemic situation may be promulgated ${ }^{18}$. Therefore, the aim of this study is to predict subsequent trend according to the epidemiological data of COVID-19, and to conduct a policy analysis to summarise meaningful and positive measures. This information has great reference significance for assessing the development of the epidemic situation in China, and in five countries with serious epidemic situations ${ }^{14}$. According to the forecast, it will be helpful to assess the effectiveness of current policies and make flexible adjustments, for instance, reinforcement, alteration, and relaxation of restrictions.

According to incomplete statistics, there are three main methods for forecasting the epidemic development of COVID19, as follows:

- The Susceptible-Exposed-Infectious-Removed (SEIR) model was used to effectively predict the peaks and sizes of COVID-19 epidemiological data with sufficient fitting performance ${ }^{14,19}$. A modified SEIR model also showed a good effect for predicting the peaks and sizes ${ }^{14}$. The peak deviation of the another modified SEIR model in predicting epidemiological data in China was $3.02 \%{ }^{19}$. However, SEIR focuses on predicting trends in sensitive, exposed, infected, and recovered groups ${ }^{14,19}$, rather than new daily cases. Moreover, it is necessary to comprehensively consider the changes in some parameter values as affected by the changes in epidemic policies and regional differences, such as the effective reproductive number, number of contacts, and infection rate.

- A modified stacked auto-encoder was used to predict the cumulative and new confirmed cases of COVID-19 in China. The average absolute error was between $0.73 \%$ and $2.27 \%$ among the cumulative confirmed cases, and the fitting of daily new cases had room for improvement ${ }^{20}$. This model involves processing a latent matrix generated by a second latent layer and forming the eigenvectors for 34 provinces and cities in China; therefore, the operation is more complicated, and the lower values in the early epidemic data will result in smaller errors.

- Long-Short-Term-Memory (LSTM) is more successful than auto-encoder for sequence processing tasks such as machine translation and speech recognition ${ }^{21}$. In COVID-19 trend prediction, LSTM has a deeper network structure and simple operations than the modified stacked auto-encoder. A neural network model based on LSTM was used to predict COVID-19 trend after pre-training using SARS data, and the results demonstrated that LSTM has good prospects for predicting the trend; however, the fitting effect needs to be improved ${ }^{14}$. A hybrid model with an LSTM was used to predict COVID-19 trends in 11 countries, and showed accurate predictive ability. The Root-Mean-Square-Error (RMSE) from the model was between 15 to 623, and we estimated that the error was between $2 \%$ and $24 \%$, except for the error of approximately $84 \%$ in China, but this model was sensitive to the large fluctuation in the data 22 .

In this study, to reduce the prediction errors and the sensitivity of daily data with large fluctuation for a better fitting effect, we train an improved three-layer superimposed LSTM (3*LSTM) neural network model based on single-layered LSTM, and use the weekly epidemiological data of China, five overseas countries and the world to predict the trend. We also use the model to analyse policy events and the social economic influences under the epidemic situation.

\section{Data sources and study area}


The study data comprised the epidemiological data of COVID-19 between January 16 and December 5, 2020 in China and the current confirmed epidemic data between January 12 and December 5, 2020 in Wuhan, Hubei Province, China, as reported by the National Health Commission of the People's Republic of China ${ }^{23}$ (PRC) and the Health Commission of Hubei Province $^{24}$. The data of the top five countries from January 22 to December 5, 2020 worldwide were sourced from a dataset based on global COVID-19 epidemic statistics, as published by Johns Hopkins University in the United States ${ }^{25}$. The five overseas countries were the United States, India, Brazil, Russia and France. The cumulative confirmed overseas epidemic data from February 15 to December 5, 2020 were retrieved from a Baidu website ${ }^{26}$. The study area is shown in the Figure 1.

We combined the Chinese and overseas COVID-19 data to generate the numbers of cumulative confirmed cases and cumulative deaths worldwide. In the process of training, we found that better prediction results could be obtained among data with more evident changes. Therefore, we took the Saturday data from the daily accumulated diagnostic data of China, the five countries, and the world. As shown in the Table 1, the start date of COVID-19 data in the study country or region varied with the start time of the epidemic; the deadline for the statistical data was December 5, 2020. Epidemiological data from China and the United States was used for multi-layer LSTM comparison and trend prediction. Data from Wuhan was used to compare the prediction effects of our LSTM model with that from SEIR models. Data from the United States, India, Brazil, Russia, France was used for comparing outbreaks in the five countries, and the global epidemiological data was used for forecast trends.

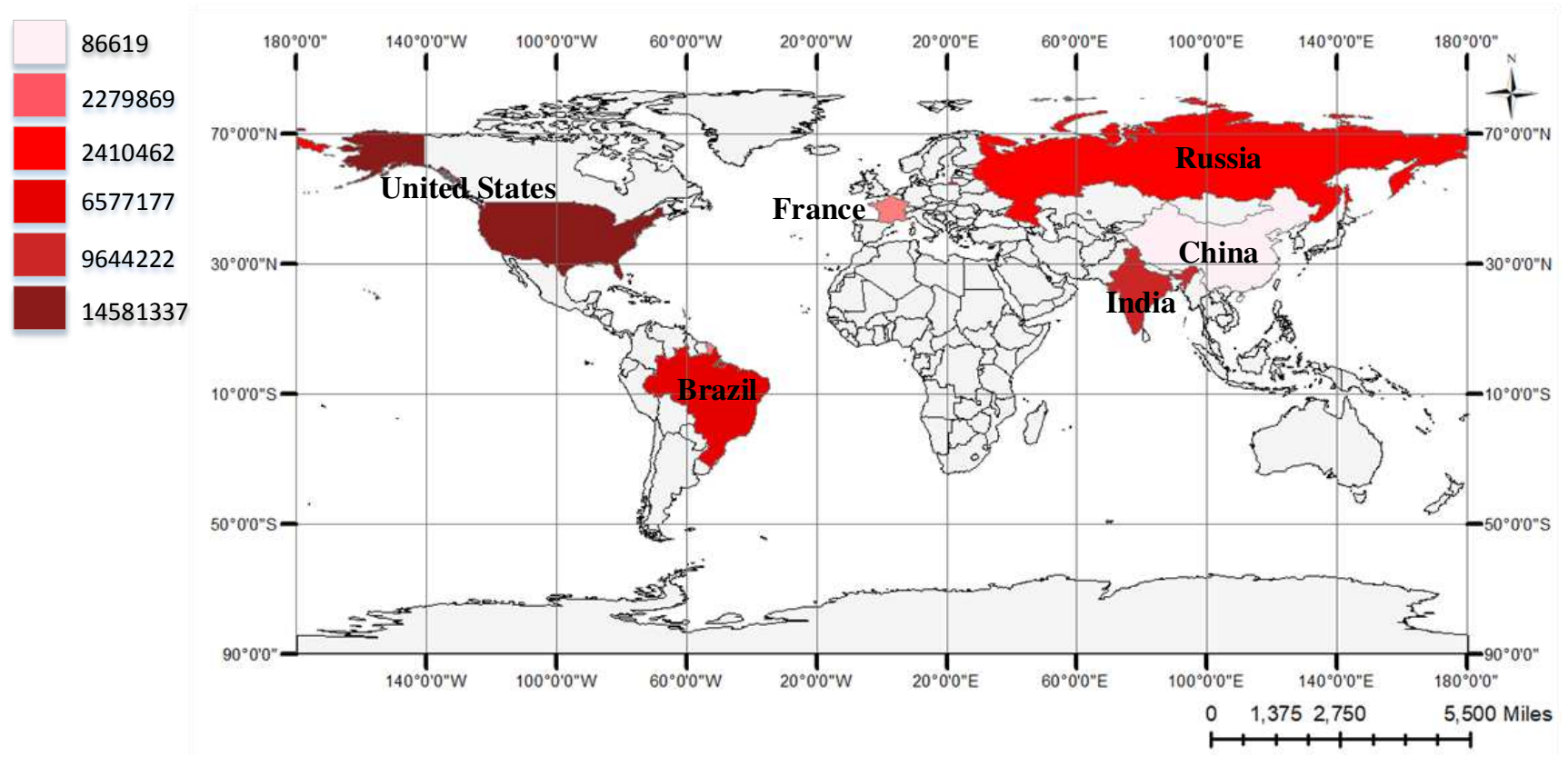

Figure 1. China and top five countries of confirmed COVID-19 cases worldwide. The data on the left represents the amount of cumulative confirmed cases in China and five countries with severe epidemic situation as of December $5,2020$. COVID-19, coronavirus disease 2019.

\begin{tabular}{|c|c|c|c|c|}
\hline Country/ Region & Confirmed & Deaths & $\begin{array}{c}\text { Cumulative cases } \\
(\mathbf{2 0 2 0 / 1 2 / 5 )}\end{array}$ & $\begin{array}{c}\text { Cumulative deaths } \\
\mathbf{( 2 0 2 0 / 1 2 / 5 )}\end{array}$ \\
\hline Global & February 15, 2020 & February 15, 2020 & 66423912 & 1527936 \\
\hline United States & January 25, 2020 & February 29, 2020 & 14581337 & 281186 \\
\hline India & February 1, 2020 & March 14, 2020 & 9644222 & 140182 \\
\hline Brazil & February 29, 2020 & March 21, 2020 & 6577177 & 176628 \\
\hline Russia & February 1, 2020 & March 21, 2020 & 2410462 & 42228 \\
\hline
\end{tabular}




\begin{tabular}{|c|c|c|c|c|}
\hline France & March 7, 2020 & March 28, 2020 & 2279869 & 54630 \\
\hline China & January 18, 2020 & January 18, 2020 & 86619 & 4634 \\
\hline
\end{tabular}

Table 1. Starting time of cumulative confirmed data and cumulative death data on countries or regions obtained from data sources, and the value of Cumulative confirmed and deaths cases as of December 5, 2020.

\section{Methods}

\section{Data processing}

Sequence Data Generation is a crucial step, and it is the premise of LSTM model training. The sequence data can reflect changes in single data in which no regularity can be traced. Therefore, the individual data were organised into sequential data. As shown in the Figure 2, the individual data dimension is $(n, 1)$, a window with a length (sequence length) of $x$ and step size of 1 was initially selected for sliding orderly through the daily data. All of the data contained in the window was considered as a data sequence, and the next day data outside the window was the corresponding label data. After the window slid through all of the daily data, the sequence data with dimension of $(n-x, x)$ was generated.

\section{Long-Short-Term-Memory (LSTM)}

LSTM is a particular type of recurrent neural network (RNN), and is mainly used to learn and overcome the long-term dependence of RNN; they both have the same basic structure ${ }^{27}$. The cyclic function in an RNN is implemented in the hidden layer, and the other two main components of the RNN are the input and output layers. Each cyclic unit has an input and output $^{21}$. Except for the last layer of cyclic units, the output of each cyclic unit and the input of the next time together determine the output of the next time ${ }^{28}$. However, too many cyclic units may lead to the loss of previously learned rule information and the existence of long-term dependence, and can lead to the problem of gradient disappearance or gradient explosion $^{21}$.

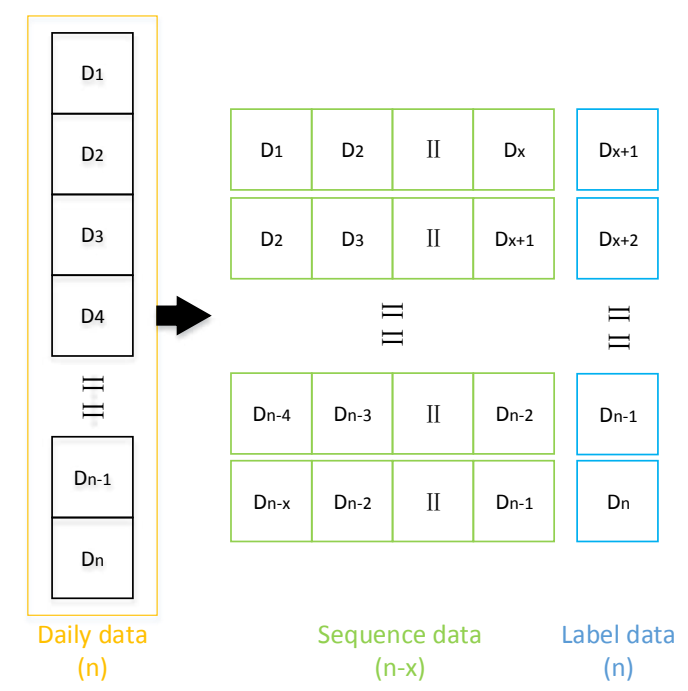

Figure 2. Sequence Data Generation

The weight value of the self-circulation introduced by LSTM is determined based on context, rather than fixed ${ }^{21}$. The internal units of LSTM include the state unit, forget gate, input gate and output gate ${ }^{21,28}$. The state unit is a core part, and information is transmitted from an upper-level unit through the state unit to the next cyclic unit after the internal update of the state unit, and the information is not affected by other internal calculations ${ }^{28}$. The sigmoid units in the gates constrain the value between 0 and 1 so as to determine which information should be saved or forgotten, thereby solving the problem of gradient disappearance, while learning long-term dependence ${ }^{21,28}$. In an LSTM cell, the first step is to discard unnecessary information through the forget gate. The second step is to determine the information stored in the state unit through the input 
gate and tanh activation layer. The input gate protects the memory information in the cyclic cell from the influences of other inputs. The final output information is determined by the output gate, and the output gate protects other cells from the memory information in the cyclic cell ${ }^{27,28}$.

In the epidemic data, the daily new case data had a certain range of continuous jitter, which led to the change of the growth rate of cumulative cases. The fitting degree of a single-layer LSTM model is low. Moreover, the prediction effect of continuous jitter data was poor and had high sensitivity.

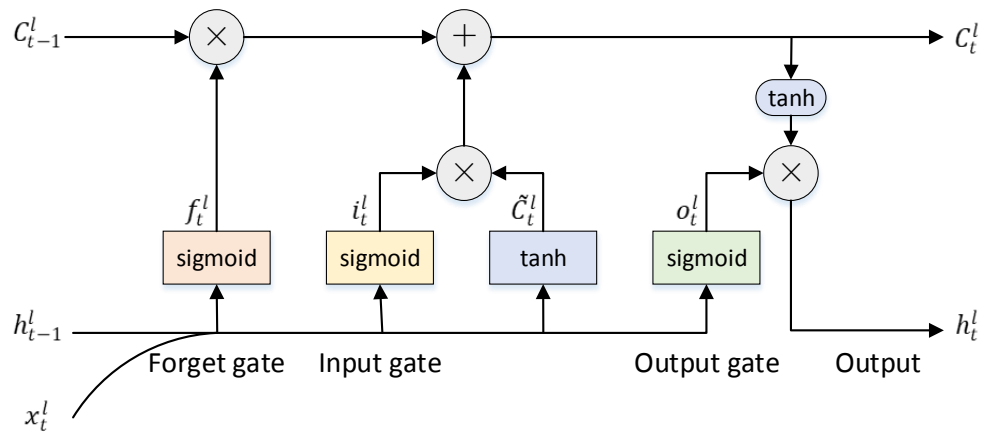

Figure 3. The inner structure of LSTM. LSTM, Long-Short-Term-Memory.

\section{Improved LSTM model}

To better train with continuously fluctuating data, fit unsmooth curves, extract regular information more effectively, and improve prediction capabilities, we deepened the number of LSTM layers. The established 3*LSTM model was used to train based on the COVID-19 epidemic data, and to predict the development trend.

In the $3 *$ LSTM model, the memory carries the changing regularity transfers between the LSTM layers and LSTM internal circulation units. When the information flow enters the circulation unit (Figure 3), the operation is as follows:

$$
\begin{gathered}
\mathrm{f}_{\mathrm{t}}^{\mathrm{l}}=\sigma\left(\mathrm{W}_{\mathrm{f}}^{\mathrm{l}} \cdot\left[\mathrm{h}_{\mathrm{t}-1}^{\mathrm{l}}, \mathrm{x}_{\mathrm{t}}^{\mathrm{l}}\right]+\mathrm{b}_{\mathrm{f}}^{\mathrm{l}}\right) \\
i_{t}^{l}=\sigma\left(W_{i}^{l} \cdot\left[h_{t-1}^{l}, x_{t}^{l}\right]+b_{i}^{l}\right) \\
\tilde{C}_{t}^{l}=\tanh \left(W_{C}^{l} \cdot\left[h_{t-1}^{l}, x_{t}^{l}\right]+b_{C}^{l}\right) \\
C_{t}^{l}=f_{t}^{l} * C_{t-1}^{l}+i_{t}^{l} * \tilde{C}_{t}^{l} \\
o_{t}^{l}=\sigma\left(W_{o}^{l} \cdot\left[h_{t-1}^{l}, x_{t}^{l}\right]+b_{o}^{l}\right) \\
h_{t}^{l}=o_{t}^{l} * \tanh \left(C_{t}^{l}\right)
\end{gathered}
$$

In the above, the LSTM layer $l=1,2,3, t$ stands for the moment, $f_{t}^{l}$, $i_{t}^{l}$ and $o_{t}^{l}$ represent the output after the activation function $\sigma$ in the forget gate, input gate and output gate, respectively. In the cell at time $t$ of LSTM in the layer $l$, the first step of information flow is to discard unnecessary information. The information matrix or vector comprising the output $h_{t-1}^{l}$ of the last moment and the input $x_{t}^{l}$ of the current moment are linearly combined with the weight $W_{f}^{l}$, and the bias offset $b_{f}^{l}$ is added. Then the linear function returns the output $f_{t}^{l}$ through the activation function $\sigma$. Second, the output $i_{t}^{l}$ of the activation layer $\sigma$ in the input gate is obtained by information matrix $\left[h_{t-1}^{l}, x_{t}^{l}\right]$, multiplied by the weight $W_{i}^{l}$, and added with the bios offset $b_{i}^{l}$. The activation function is used to determine which information needs to be saved or updated, and then a vector containing the updated information $\tilde{C}_{t}$ is generated through the tanh activation layer. The updated cell state $C_{t}$ is obtained by adding $f_{t}^{l} * C_{t-1}^{l}$ which represents unnecessary information to be discarded, and $i_{t}^{l} * \tilde{C}_{t}^{l}$ which represents the information to be updated. Finally, whether the information will be output is selected by the activation layer $\sigma$. The information value $\tanh \left(C_{t}^{l}\right)$ (in the range -1 to 1 after $\tanh$ layer) is obtained and multiplied by the output $o_{t}^{l}$ of active layer to return the final output $h_{t}^{l}$ for this LSTM cell. 
As shown in Figure $4, x_{0}, x_{1}, \ldots, x_{t}$ represents sequence data generated from individual data. The three different coloured layers represent the expanded cyclic units of the three LSTM layers. Each LSTM cell has an input $x$ and output $h$. The final output of the upper LSTM layer is used as the input to the next LSTM layer. The output of the first LSTM layer, and second LSTM layer is $\left[h_{0}^{l}, h_{1}^{l}, \ldots, h_{t}^{l}\right],(l=1,2)$, which represents the set of outputs from each cyclic unit in that layer. The third LSTM layer outputs the final $h_{t}^{3}$, i.e. the output value of the last cyclic unit of the layer. Then it determines the dimension to be output through the dense layer. Finally, the linear activation layer is used to fit the output data and label data to further reduce the error. The output data of the model continues to be trained to predict data trends.

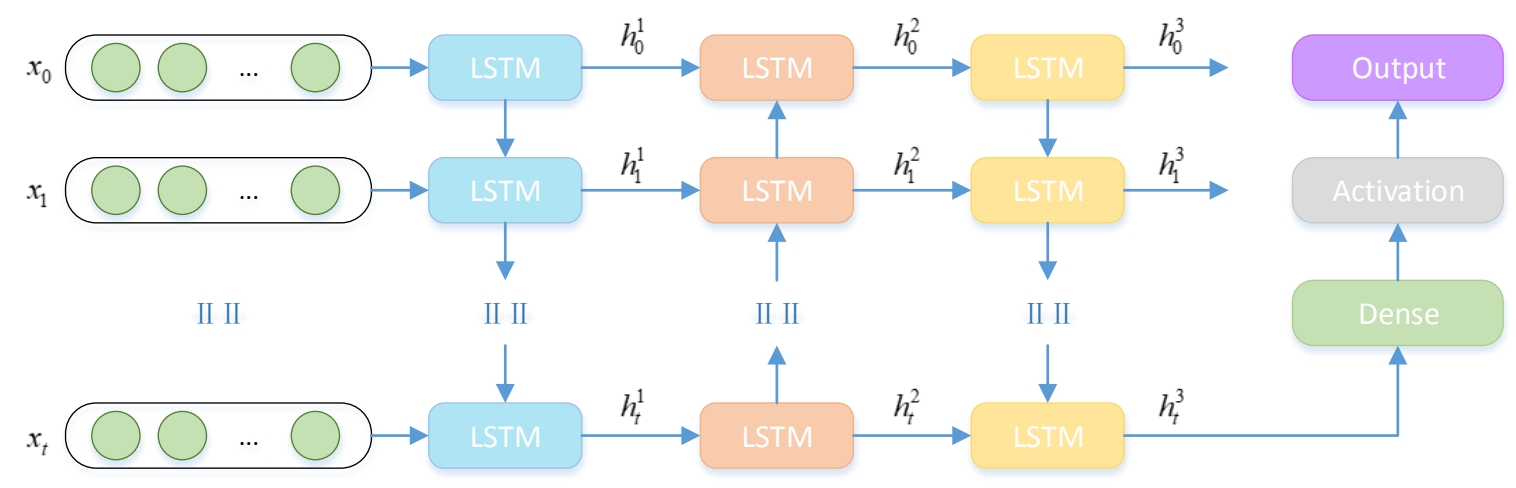

Figure 4. LSTM model of recurrent expansion. $x$ indicates the sequence data, $h$ represents the output of the LSTM recurrent unit. The blue is the first LSTM layer, orange is the second LSTM layer, and yellow is the third LSTM layer. LSTM, Long-Short-Term-Memory.

\section{Assessment metrics}

To evaluate the effect of our model, the RMSE was used to represent the fitting degree between the output (prediction data) and label data of each sequence data after $3 *$ LSTM model training. The RMSE function is as follows:

$$
\operatorname{RMSE}(L, h)=\sqrt{\frac{1}{m} \sum_{i=1}^{m}\left(L_{i}-h_{i}\right)^{2}}
$$

In the LSTM Model, $m$ denotes the number of sequential data, and $h_{i}$ and $L_{i}$ represent the output of each sequence $i$ after LSTM training and the corresponding label data, respectively. The smaller the value of RMSE, the better the prediction effect, which means that the error between prediction data and label data is smaller.

\section{Results}

\section{Training environment}

The deep learning environment of our experiment was mainly built based on the Ubuntu system environment, which combines Keras and Tensorflow for training with the support of a GPU. The detailed parameters are showed in Table 2.

\begin{tabular}{|c|c|}
\hline Environment & Version \\
\hline GPU & GeForce RTX 2080 Ti/PCle/SSE2 \\
\hline Ubuntu & 16.04 LTS \\
\hline Python & 3.5 .2 \\
\hline Tensorflow & $1.12 .0-\mathrm{rc} 2$ \\
\hline Keras & 2.2 .4 \\
\hline Pandas & 0.24 .2 \\
\hline Sklearn & 0.00 .2$. post1 \\
\hline
\end{tabular}

Table 2. Version of hardware and software in deep learning environment. 


\section{Comparison of LSTM models with different layers}

We used the COVID-19 weekly confirmed case data from China and the United States to evaluate the LSTM model. To compare the prediction effects from the different amount of LSTM layers, we trained these networks with the time steps of $3,5,7,9$ and 11 weeks. The error rate of these models exceeds ' 1 ' when the time step is 3 or 5 weeks. Therefore, we do not show these results. Table 3 lists the RMSE values under the model structures with different numbers of LSTM layers with 3000 epochs. The running time of an epoch increases with the number of stacking layers of the LSTM, along with the data length. Table 3 indicates that the stability of $3 *$ LSTM and $4 *$ LSTM models are generally superior to that of $1 *$ LSTM and $2 *$ LSTM. However, $4 *$ LSTM model is more time-consuming. Hence, we mainly employed $3 *$ LSTM model for the rest of the experiment.

\begin{tabular}{|c|c|c|c|c|c|c|}
\hline Country & Model & Error & 7 weeks & 9 weeks & 11 weeks & Time(minutes) \\
\hline \multirow{8}{*}{ China } & \multirow{2}{*}{$1 * \mathrm{LSTM}$} & RMSE & 697.97 & 0.803 & 281.45 & \multirow{2}{*}{25.60} \\
\hline & & Error rate & $0.78 \%$ & $0.53 \%$ & $0.27 \%$ & \\
\hline & \multirow{2}{*}{$2 *$ LSTM } & RMSE & 302.81 & 287.21 & 278.44 & \multirow{2}{*}{40.43} \\
\hline & & Error rate & $0.29 \%$ & $0.28 \%$ & $0.29 \%$ & \\
\hline & \multirow{2}{*}{$3 *$ LSTM } & RMSE & 418.67 & 289.92 & 208.28 & \multirow{2}{*}{55.51} \\
\hline & & Error rate & $0.44 \%$ & $0.29 \%$ & $0.19 \%$ & \\
\hline & \multirow{2}{*}{$4 *$ LSTM } & RMSE & 660.74 & 235.12 & 288.23 & \multirow{2}{*}{68.56} \\
\hline & & Error rate & $0.73 \%$ & $0.21 \%$ & $0.27 \%$ & \\
\hline \multirow{8}{*}{ US } & \multirow{2}{*}{$1 *$ LSTM } & RMSE & 50090.84 & 31317.14 & 42306.43 & \multirow{2}{*}{24.59} \\
\hline & & Error rate & $37.06 \%$ & $2.48 \%$ & $0.60 \%$ & \\
\hline & \multirow{2}{*}{$2 *$ LSTM } & RMSE & 106721.87 & 58988.65 & 59088.7 & \multirow{2}{*}{39.06} \\
\hline & & Error rate & $114.72 \%$ & $2.96 \%$ & $0.85 \%$ & \\
\hline & \multirow{2}{*}{$3 *$ LSTM } & RMSE & 40879.84 & 38332.94 & 65520.27 & \multirow{2}{*}{53.22} \\
\hline & & Error rate & $68.16 \%$ & $2.66 \%$ & $1.20 \%$ & \\
\hline & \multirow{2}{*}{$4 *$ LSTM } & RMSE & 55511.05 & 89176.01 & 33590.64 & \multirow{2}{*}{69.66} \\
\hline & & Error rate & $80.30 \%$ & $6.02 \%$ & $1.06 \%$ & \\
\hline
\end{tabular}

Table 3. The RMSE of LSTM model comparison. Prediction of cumulative confirmed COVID-19 cases in China and the United States. The time steps of 7, 9 and 11 weeks are used in the model. RMSE, Root-Mean-Square-Error; LSTM, LongShort-Term-Memory. COVID-19, coronavirus disease 2019.

\section{Comparison of 3*LSTM model and Susceptible-Exposed-Infectious-Removed (SEIR) model}

We used current confirmed cases in Wuhan from February 12, 2020 to December 5, 2020, and the forecast date ended on January 16, 2021. The current confirmed cases in Wuhan refer to confirmed cases excluding deaths and rehabilitated people, and we regard it as the same parameter as the 'infected people' parameter in the SEIR model. We took $3 *$ LSTM model for training with a time step of 33 days and 3000 epochs, and the RMSE was 30.49 . 


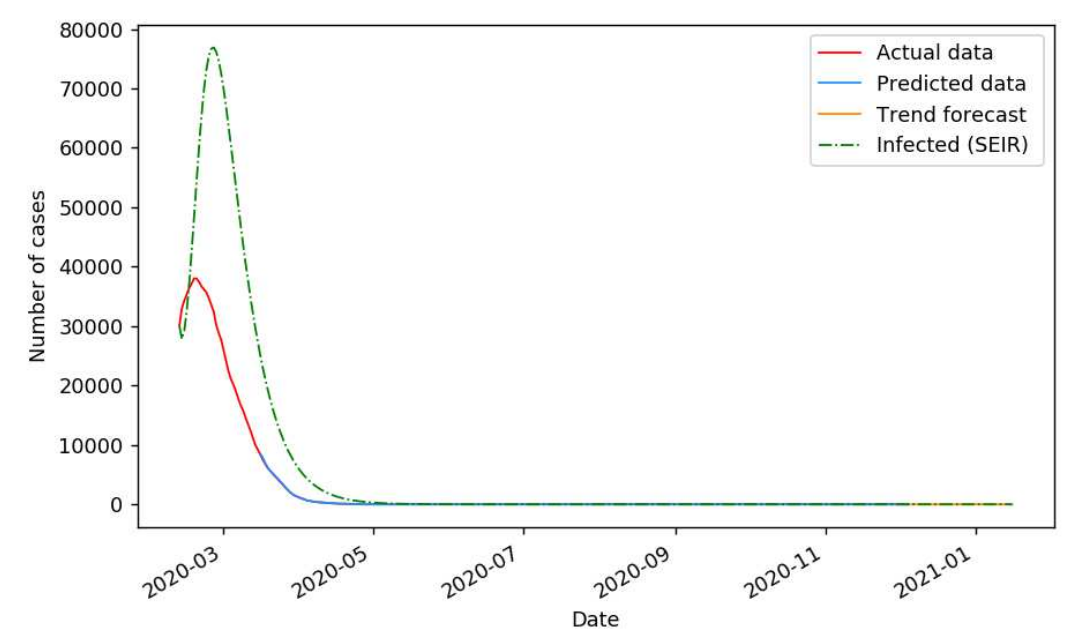

Figure 5. Comparison of $3 *$ LSTM model and SEIR model ${ }^{19}$ in the current confirmed COVID-19 cases of Wuhan, Hubei Province, China. The solid red line is real data, the solid blue line represents the predicted data with label data training in the LSTM model, the solid green line indicates the trend prediction in the LSTM model, and the dashed orange line denotes the number of infected individuals as predicted by the SEIR model. LSTM, Long-Short-Term-Memory; SEIR, SusceptibleExposed-Infectious-Removed. COVID-19, coronavirus disease 2019.

According to the SEIR model, the population in Wuhan, Hubei Province, China is divided into four groups based on disease stage: susceptible $(\mathrm{S})$, exposed $(\mathrm{E})$, infected $(\mathrm{I})$, and recovery $(\mathrm{R})^{19}$. As Wuhan inhabitants took corresponding prevention and control measures after the closure of the city, not all residents are at risk of infection, so the total number of potential contacts used was $N=200000^{29}$. Based on the report of National Health Commission, PRC on February 12, 2020, the quantities of infected, exposure and recovery individuals before running the SEIR model ${ }^{19}$ were $I_{0}=30043, E_{0}=$ 426 , and $R_{0}=2951$, respectively.

Figure 5 shows that the values predicted by $3 *$ LSTM model and actual values are basically coincident, whereas the predicted values from SEIR model are higher than the actual values from February to May. In the trend forecast stage, the predicted values from $3 *$ LSTM model are between 2 and 3, and the SEIR-infected people of are predicted to drop to 0 after July 3. In the SEIR model, the parameter values (such as infection rate, frequencies of exposure, and contacts) are affected by the measures taken or adjusted in different periods, as well as the values of the four groups and total quantity. This results in a large difference between the predicted and actual values.

\section{Comparison of the epidemic situations between China and the US}

The first COVID-19 case of in China began to show symptoms on December 1, 2019 ${ }^{30}$. After the outbreak, the Chinese government issued a timely emergency response: laboratories expanded their capacities for nucleic acid detection, the 'Huoshenshan', 'Leishenshan', and Fangcang shelter hospitals were established for accommodating more patients, medical observation was conducted on close contacts, the source of infection was investigated, people remained at home as much as possible, and wearing masks, avoiding gatherings, and keeping a physical distance of 1 meter when going out were strictly required ${ }^{17,31}$. The China CDC issued prevention guidelines for people of different ages and places to strengthen the awareness of safety $^{32}$. Under the guidance of national policies and the common resistance of people across the country, the epidemic has been promptly controlled, and the number of confirmed cases has gradually decreased. The epidemic situation has entered the stage of continuous prevention and control, and the resumption of work and school has been flexibly promoted ${ }^{33}$.

On January 20, 2020, the United States recorded its first COVID-19 patient ${ }^{34}$. The initial measure after the outbreak in the United States was to temporarily ban entry of non-Americans who had visited China in the past 14 days ${ }^{35}$. However, the 
number of infected people increased, owing to flows of people at home and abroad ${ }^{36}$. The CDC proposed that residents wear masks in public places to reduce transmission on April $3^{37}$, but many people ignored this advice, and previously unknown challenges existed, owing to a campaign of mass protests and campaign rallies without protective measures ${ }^{3636}$. In addition, the 'opening up' measures promoted by the American government led to increases in the quantity of hospitalizations ${ }^{38}$. As a result, the account of confirmed cases in the United States continued to increase significantly after surpassing China on March 26, 2020. The general development stages of the epidemic in China and the United States are summarized in the Table 4 and Table 5.

We used cumulative data from China and the United States for training. Owing to the lack of weekly data, and to better modeling effects, we used odd numbers from 3 to 35 weeks as the time steps for independent training. The optimal prediction effect was obtained when the time step of cumulative cases was 33 weeks. As shown in Figure 6a, the epidemic in China has subsided since March, the amount of daily new confirmed cases has gradually been maintained below 50. The COVID-19 deaths in China have been basically flat since late April and have remained at 4634 since May 6, and the weekly mortality rate has dropped to 0 (Error! Reference source not found.b, Figure 7). In Figure 7b, 1290 death cases were increased after data revision in mid-April, 2020, resulting in abnormal peak value. The quantity of cumulative confirmed cases in the United States has been rising sharply after surpassing China on March 26, 2020, the growth rate has not shown a decline, and deaths in the United States are increasing by thousands a week (Figure 8). The weekly mortality rate in the United States has surpassed that of China since late April, 2020; fortunately, the mortality has shown a downward trend (Figure 9). In particular, mortality in the United States has gradually declined after reaching its maximum in May; thia may be related to the policy of wearing masks. The data in Table 6 shows that the difference between the predicted and the actual values is less than $0.4 \%$, indicating that the model has good predictive fitting performance.

\begin{tabular}{|c|c|c|}
\hline Date $^{33}$ & Stage $^{33}$ & Event \\
\hline $\begin{array}{l}\text { Early December to mid- } \\
\text { December } 2019\end{array}$ & Occurrence stage & $\begin{array}{l}\text { On December 1, 2019, the first confirmed case of COVID-19 } \\
\text { developed symptoms }{ }^{30} \text {. }\end{array}$ \\
\hline $\begin{array}{l}\text { Late December } 2019 \text { to } \\
\text { mid-January } 2020\end{array}$ & Development stage & $\begin{array}{l}\text { (1) From December } 26 \text { to December } 29,2019,7 \text { patients } \\
\text { with lung abnormalities appeared successively }{ }^{39} \text {; } \\
\text { (2) Person-to-person transmission }{ }^{13} \text {; } \\
\text { (3) China CDC issued emergency response }{ }^{13} \text {. } \\
\text { (4) The number of cases increased, and the epidemic } \\
\text { spread to other parts of China }{ }^{23} \text {. }\end{array}$ \\
\hline $\begin{array}{l}\text { Late January to early March } \\
\qquad 2020\end{array}$ & Explosive stage & $\begin{array}{l}\text { (1) At } 10 \text { o'clock on January } 23,2020 \text {, Wuhan was closed, } \\
\text { and other areas were closed }{ }^{40} \text {; } \\
\text { (2) Initiation successive of first level response in various } \\
\text { regions across the country }{ }^{41} \text {; } \\
\text { (3) "Huoshenshan", "Leishenshan", Fangcang shelter } \\
\text { hospitals in Wuhan were completed }{ }^{42} \text {; } \\
\text { (4) February 1, Prevention and control guidelines, Edition } \\
1^{32} \text {. }\end{array}$ \\
\hline $\begin{array}{c}\text { Mid-March } 2020 \text { to April } \\
28,2020\end{array}$ & Recession stage & $\begin{array}{l}\text { (1) The incident was under basic control; } \\
\text { (2) Regions began to unseal, Wuhan officially unblocked on } \\
\text { April } 8,2020^{43} \text {; }\end{array}$ \\
\hline
\end{tabular}




\begin{tabular}{|c|c|c|}
\hline $\begin{array}{l}\text { April } 29 \text { to December 5, } \\
\qquad 2020\end{array}$ & $\begin{array}{c}\text { Continuous prevention } \\
\text { and control }\end{array}$ & $\begin{array}{l}\text { (1) As of May 2, 2020, all provinces across the country } \\
\text { have lifted the first-level emergency response } \\
\text { (2) The promotion of resumption of work and school }{ }^{33} \text {. }\end{array}$ \\
\hline & & \\
\hline
\end{tabular}

Table 4. Basic development of the epidemic situation in China. Different strategies or events of China at different stages of the COVID-19 epidemic. COVID-19, coronavirus disease 2019.

\section{Top five countries in the global cumulative diagnosis}

The weekly cumulative confirmed case data from five countries are trained through the $3 *$ LSTM model. These five countries are the US, India, Brazil, Russia, and France. From the perspective of epidemic prevention and control, in some countries, measures were not strictly enforced, or the severity of the epidemic was ignored so as to restart the economy (e.g. the early release of blockade restrictions in some states of the United State $)^{52}$. Brazil implemented a social distancing policy, but some people neglected to wear masks at rallies ${ }^{53}$, and professional football matches were held in Rio, Brazil on June 18, $2020^{54}$. The Indian government implemented the 'city closure' measures at the end of March, but some areas have been re-blocked, because of an outbreak rebound caused by the increase of outdoor activities after a gradual unsealing ${ }^{55,56}$. The Russian government did not take timely measures to prevent the European epidemic, causing a large number of imported cases ${ }^{57}$; moreover, a military parade was held on June $24^{58}$. France implemented a lockdown on March 17, 2020, and gradually unblocked it from May 11, 2020. However, the French government closed cities for a second time on October 30 after the emergence of cases in school and surges in cases in many areas ${ }^{59,60}$.

\begin{tabular}{|c|c|c|}
\hline $\operatorname{ate}^{36}$ & Stage & Event \\
\hline $\begin{array}{l}\text { January } 20,2020 \text { to } \\
\text { February } 23,2020\end{array}$ & Occurrence stage & $\begin{array}{l}\text { (1) On January 20, 2020, the first confirmed case was reported }{ }^{34} \text {; } \\
\text { (2) During this time period, the US confirmed } 14 \text { local cases and } 39 \\
\text { cases were withdrawn from overseas }{ }^{45} \text {. } \\
\text { (3) At 5:00 pm on February 2nd, Eastern Time, foreigners who have } \\
\text { been to China in the past } 14 \text { days will be provisionally } \\
\text { prohibited entry, while American citizens will be quarantined } \\
\text { for } 14 \text { days }^{35} \text {. }\end{array}$ \\
\hline $\begin{array}{l}\text { Late February } 2020 \text { to } \\
\text { early March } 2020\end{array}$ & Development stage & $\begin{array}{l}\text { (1) Cases of travel history in epidemic-free areas appeared, the } \\
\text { epidemic was gradually spreading in the US } S^{45} \text {; } \\
\text { (2) Mardi Gras celebrations, International professional conference } \\
\text { in Boston, the funeral in Albany }{ }^{36} \text {; } \\
\text { (3) On March } 14, \text { Cruise ship ban }{ }^{36} \text {. }\end{array}$ \\
\hline $\begin{array}{l}\text { Mid-March } 2020 \text { to } \\
\text { December 5, } 2020\end{array}$ & Explosive stage & $\begin{array}{l}\text { (1) On April 3, the US CDC required residents to wear masks in } \\
\text { public places } 37 \text {; } \\
\text { (2) "Black Lives Matter" protest in parts of the US }{ }^{46} \text {; } \\
\text { (3) US President Trump held the first campaign rally in Tulsa }{ }^{47} \text {; } \\
\text { (4) More U.S. states demand wearing masks in public }{ }^{48} \text {; } \\
\text { (5) Trump called for wearing masks }{ }^{49} \text {; } \\
\text { (6) Trump pushed reopening }{ }^{38} \text {; } \\
\text { (7) In late August, many schools opened }{ }^{50} \text {. } \\
\text { (8) In October, } 34 \text { people diagnosed in the White } \text { House }^{51} \text {. }\end{array}$ \\
\hline
\end{tabular}

Table 5. Basic development of the epidemic situation in the United States. Different strategies or events of the United States at different stages of the COVID-19 epidemic. COVID-19, coronavirus disease 2019. 


\begin{tabular}{|c|c|c|c|}
\hline Country & Data & RMSE & Error rate \\
\hline \multirow{2}{*}{ China } & Cumulative cases & 396.8 & $0.39 \%$ \\
\cline { 2 - 4 } & Cumulative deaths & 7.86 & $0.17 \%$ \\
\hline
\end{tabular}

Table 6. Prediction of cumulative COVID-19 cases in China by $3 *$ LSTM model. The error between the predicted value and the true value is expressed as a percentage. LSTM, Long-Short-Term-Memory; COVID-19, coronavirus disease 2019.

a

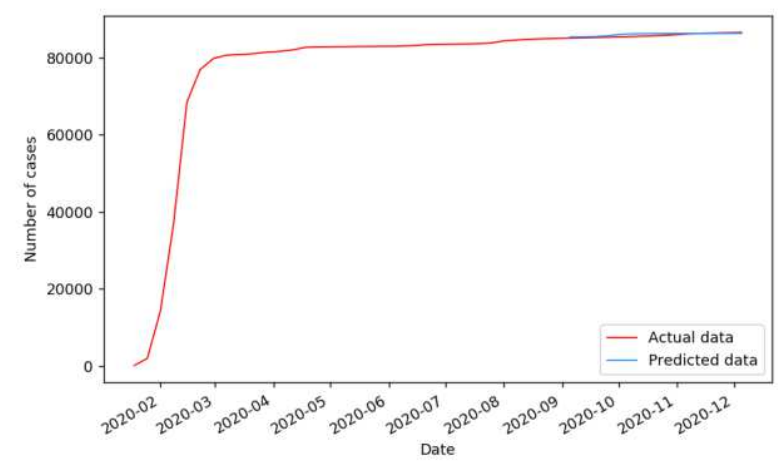

b

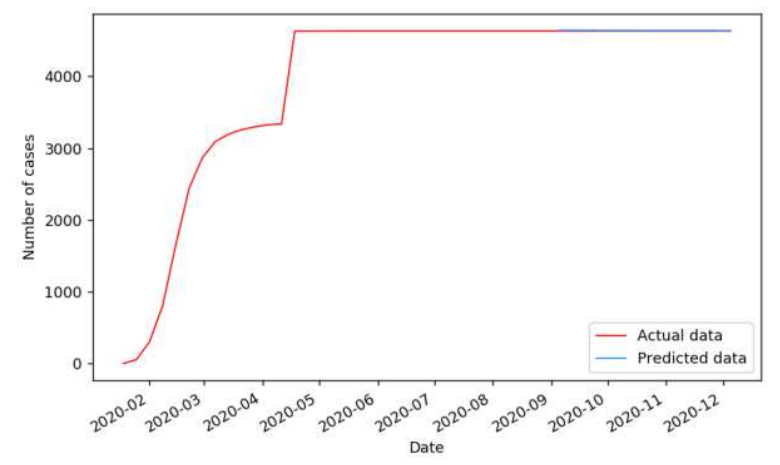

Figure 6. Prediction of cumulative COVID-19 cases in China and the United States by $3 *$ LSTM model. (a) Cumulative confirmed cases in China. (b) Cumulative deaths in China. LSTM, Long-Short-Term-Memory. COVID-19, coronavirus disease 2019.

a

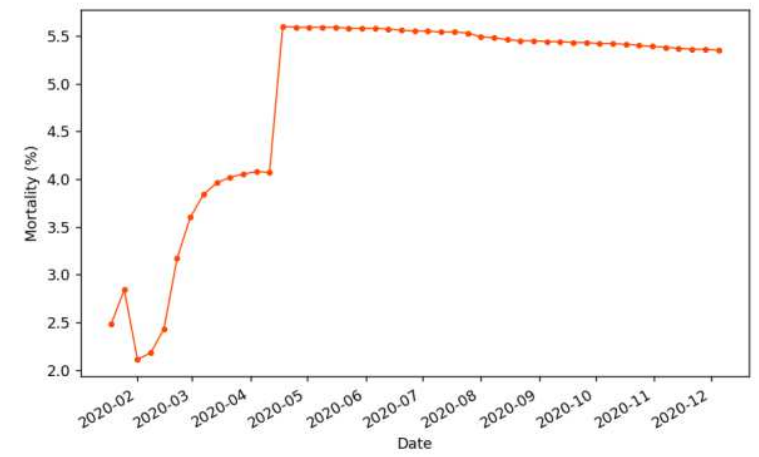

b

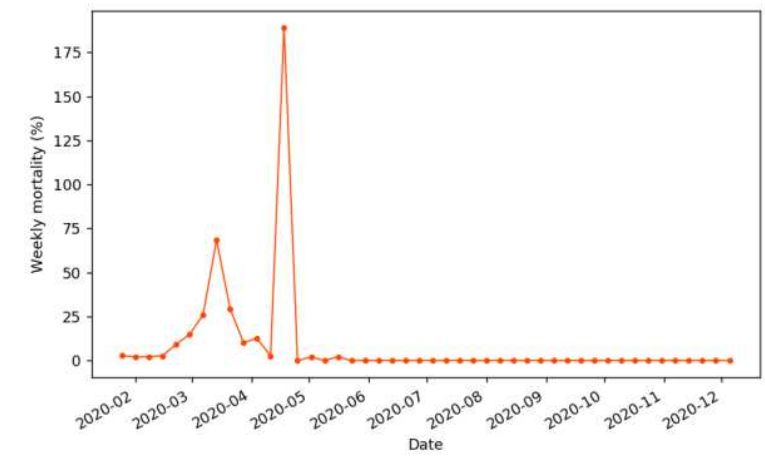

Figure 7. The mortality of COVID-19 cases in China and the United States. (a) Cumulative mortality. (b) Weekly mortality: the rate of weekly new deaths and new cases. COVID-19, coronavirus disease 2019. 
a

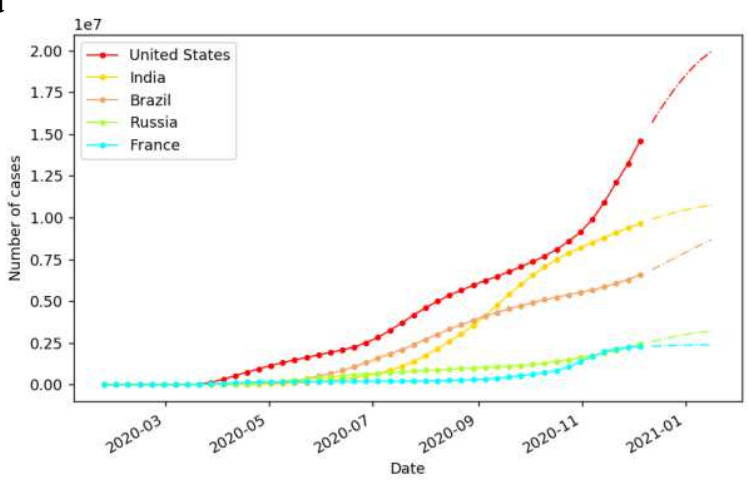

b

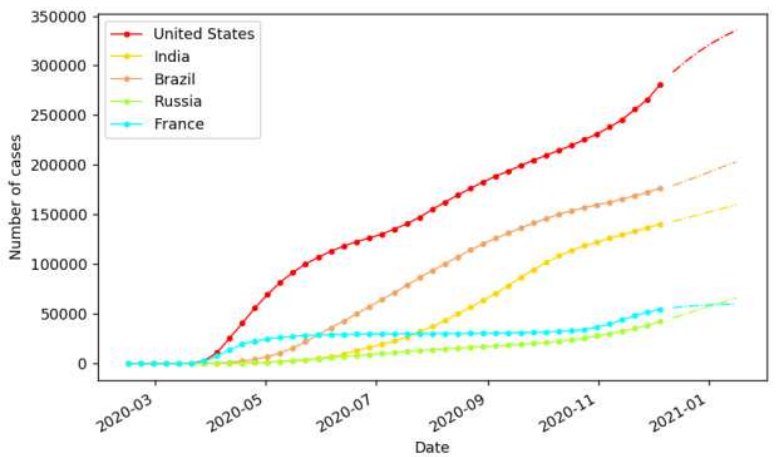

Figure 8. Prediction of cumulative COVID-19 cases in five countries by $3 *$ LSTM model. (a) Prediction of weekly cumulative confirmed cases. (b) Prediction of weekly cumulative death cases. The solid line is the actual data, and the dotted line is the forecast data. LSTM, Long-Short-Term-Memory; COVID-19, coronavirus disease 2019.

\begin{tabular}{|c|c|c|c|c|}
\hline \multicolumn{1}{|c|}{ Data } & Country & RMSE & Error rate & Prediction (2021/1/16) \\
\hline \multirow{4}{*}{ Cumulative cases } & United States & 40315.80 & $0.31 \%$ & 19962492 \\
\cline { 2 - 5 } & India & 18125.20 & $0.17 \%$ & 10745763 \\
\cline { 2 - 5 } & Brazil & 10963.08 & $0.18 \%$ & 8675770 \\
\cline { 2 - 5 } & Russia & 11269.80 & $0.54 \%$ & 3196439 \\
\cline { 2 - 5 } & France & 19481.06 & $1.36 \%$ & 2382998 \\
\hline \multirow{3}{*}{ Cumulative deaths } & United States & 1841.25 & $0.69 \%$ & 335520 \\
\cline { 2 - 5 } & India & 614.79 & $0.47 \%$ & 159801 \\
\cline { 2 - 5 } & Brazil & 1253.58 & $0.74 \%$ & 203261 \\
\cline { 2 - 5 } & Russia & 8.7 & $0.02 \%$ & 66022 \\
\hline
\end{tabular}

Table 7. Prediction of cumulative confirmed and death COVID-19 cases in five countries by $3 *$ LSTM model. RMSE, RootMean-square-Error; LSTM, Long-Short-Term-Memory; COVID-19, coronavirus disease 2019.

a

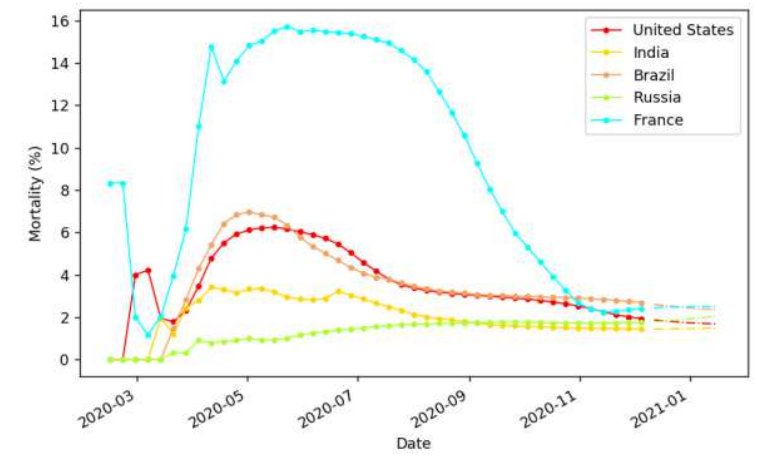

b

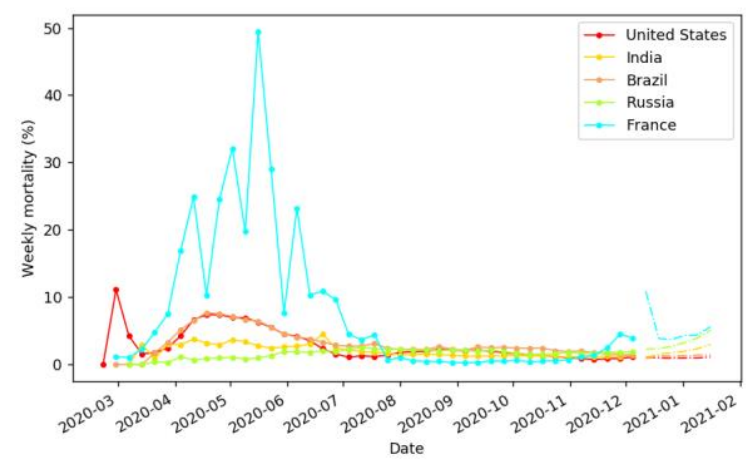

Figure 9. Mortality comparison of COVID-19 patients in five countries. (a) Cumulative mortality. (b) Weekly mortality: the rate of weekly new deaths and new cases. The solid line represents the actual mortality, and the dotted line represents the predicted mortality by $3 *$ LSTM model. COVID-19, coronavirus disease 2019; LSTM, Long-Short-Term-Memory.

The time steps used for cumulative confirmed cases and deaths were 33. Figure 8 shows the forecasts for the five countries. 
Except for France, the cumulative confirmed cases and deaths of the other four countries have no evident flattening trend. Russia has more cumulative confirmed cases and fewer deaths than France, but the numbers of cumulative deaths is expected to exceed France in early January, 2021. In Table 7, the error rate between predicted and actual values is less than $1.4 \%$. According to the forecast, the cumulative cases of the worst outbreak (in the United States) will exceed 17.8 million by the end of 2020 and 19.9 million in mid-January, 2021. The epidemic situations in the US, India and Brazil remain high-risk. Figure 9 shows the cumulative and weekly mortality rates, based on actual and predicted cumulative confirmed cases and deaths in the five countries. The cumulative mortality rate in India, Russia and France is expected to maintain an increase within $0.1 \%$, whereas those in the United States and Brazil are estimated to decline slowly. The continuously declining weekly mortality rate in the United States may rise after 3 weeks. The weekly mortality rate in the other four countries is forecast to rise slightly. It is expected that the higher weekly mortality rate in France next week may be caused by lower number of new confirmed cases. In addition, the weekly mortality rate in France may reach 5.6\% on the third Saturday of January, 2021.

\section{Trend prediction of global diagnosis}

The $3 *$ LSTM model was used to predict epidemic trends by training the cumulative confirmed cases for the global week from February 15 to December 5, 2020. Table 8 shows the results from model fitting and prediction, and Figure 10 displays the prediction generation diagram. According to the forecast, the quantity of cumulative diagnoses worldwide continues to rise, but the speed will gradually slow down. By January 16, 2021, the number of global cumulative confirmed cases may surpass 84.58 million, and deaths may reach 1.85 million. In Figure 11, we calculate the mortality of COVID-19, based on actual and predicted cumulative confirmed cases and deaths. The mortality of global COVID-19 patients has been declining after reaching a peak $(7.12 \%)$ in May, and this trend is expected to continue. Nevertheless, the weekly mortality rate may rise.

\begin{tabular}{|c|c|c|c|c|}
\hline \multirow{2}{*}{ Region } & cases & RMSE & Error rate & Prediction (2021/1/16) \\
\hline \multirow{2}{*}{ Global } & Cumulative cases & 97164.12 & $0.17 \%$ & 84581500 \\
\cline { 2 - 5 } & Cumulative deaths & 2230.98 & $0.17 \%$ & 1852826 \\
\hline
\end{tabular}

Table 8. Prediction of cumulative COVID-19 cases worldwide by $3 *$ LSTM model. COVID-19, coronavirus disease 2019; LSTM, Long-Short-Term-Memory.

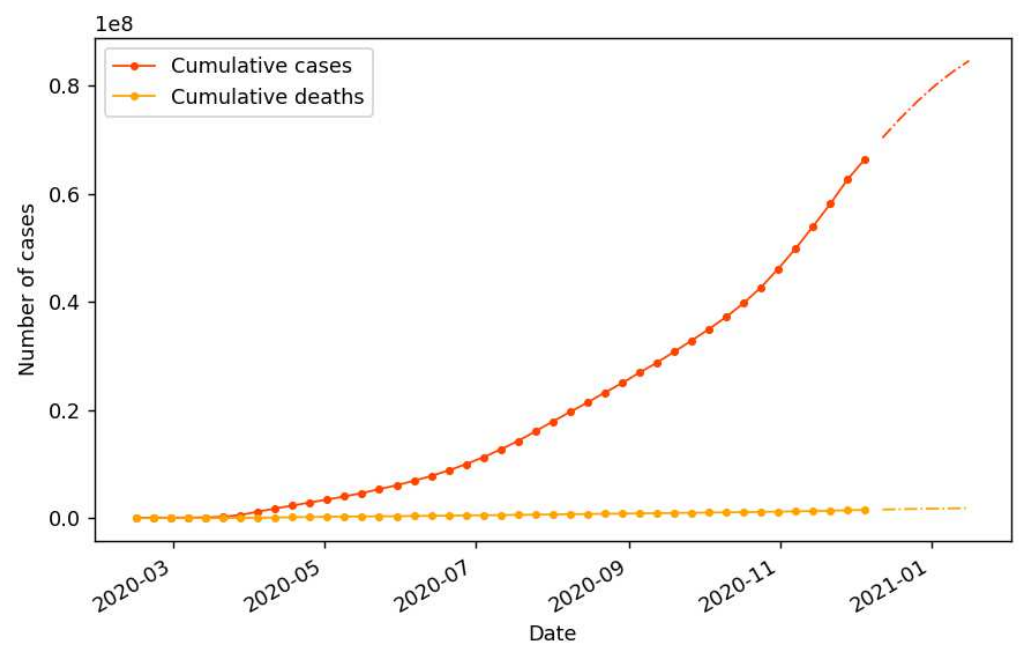

Figure 10. Prediction of global cumulative COVID-19 cases by $3 *$ LSTM model. The solid line is actual data, the dotted line represents the predicted data. LSTM, Long-Short-Term-Memory; COVID-19, coronavirus disease 2019. 
a

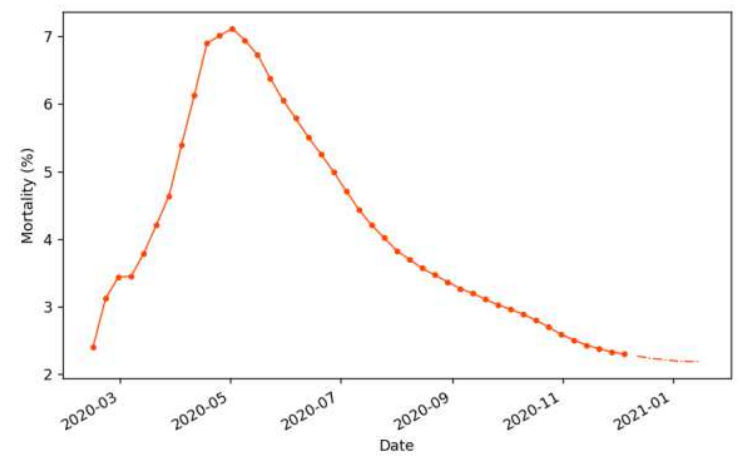

b

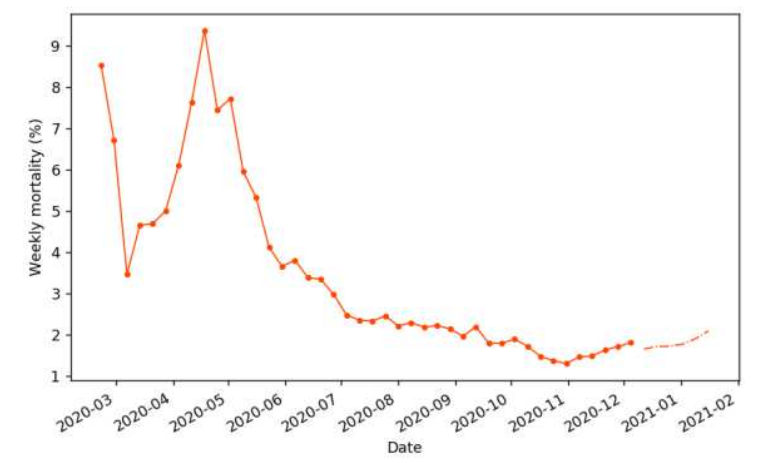

Figure 11. Forecast of global COVID-19 mortality. (a) Cumulative mortality. (b) Weekly mortality: the rate of weekly new deaths and new cases. The solid line is actual data, the dotted line represents the predicted data. COVID-19, coronavirus disease 2019 .

\section{Discussion}

According to the experimental results, it can be considered that the LSTM model can understand the regular pattern of epidemic data, has good learning capabilities, and fits training data more accurately. The prediction accuracy of $3 *$ LSTM model is better than that of the other LSTM models in this study. Our model shows that the error rate of China, five countries and the world is less than $1.4 \%$. The fitting degree of the $3 *$ LSTM model is better than the that of SEIR model among the COVID-19 data from Wuhan, and the 3 *LSTM model can combine multiple real-time data for prediction. In addition, the model applies the rules learned from the training data in the trend prediction stage, especially the cumulative case data of the top five countries and the world, and the predicted results conform to the general trends shown by the data.

The high numbers of the cases, frequent changes in the slope of the curve, and the scarcity of training data will affect the fit of the model. This is an abnormal phenomenon in that when cumulative confirmed cases are predicted in a longer time step, the predicted value is consistent with the true value, but the predicted value decreases during the trend forecast phase. One possibility is that the model overly emphasises the decreasing trend of slope value when modelling the gradually gentle data curve, causing the prediction curve to decline. Although our model performs well for data fitting, it is only a forecast for the current policy situation, and cannot predict sudden increases in data at a later stage owing to policy easing or other activities. Thus, the prediction results of the model are for reference only.

\begin{tabular}{|c|l|}
\hline Type & \multicolumn{1}{|c|}{ Event } \\
\hline \multirow{5}{*}{ positive } & (1) Release of emergency response; \\
& (2) Seal off the city, close entertainment venues; \\
(3) The prohibition of activities; \\
(4) Establishment of temporary hospital; \\
(5) Border inspection; \\
(6) Cruise ship ban; \\
(7) Prevention and control guidelines. \\
(8) Vigorously produce medical materials, improvement of the detection ability of \\
\\
(1) Relax restrictions too early for opening; \\
(2) Policy implementation is not timely; \\
(3) Hold great events: demonstration, campaign, festival; \\
(4) Face Masks are not mandatory.
\end{tabular}

Table 9. Positive and negative events. A summary based on Table 4. Basic development of the epidemic situation in China. 
Different strategies or events of China at different stages of the COVID-19 epidemic. COVID-19, coronavirus disease 2019. , Table 5. Basic development of the epidemic situation in the United States. Different strategies or events of the United States at different stages of the COVID-19 epidemic. COVID-19, coronavirus disease 2019.

\begin{tabular}{|c|c|c|c|}
\hline Country & Data & RMSE & Error rate \\
\hline \multirow{2}{*}{ China } & Cumulative cases & 396.8 & $0.39 \%$ \\
\cline { 2 - 4 } & Cumulative deaths & 7.86 & $0.17 \%$ \\
\hline
\end{tabular}

Table 6. Prediction of cumulative COVID-19 cases in China by $3 *$ LSTM model. The error between the predicted value and the true value is expressed as a percentage. LSTM, Long-Short-Term-Memory; COVID-19, coronavirus disease 2019.

, and events in ten severely affected countries.

Based on summarizing positive and negative measures during the epidemic (Table 9), we conclude that the timely release of prevention and control policies by the government is the most important. The movement and exposure of people within a country should be reduced by sealing off cities, closing entertainment venues and the prohibiting activities. The establishment of border inspection and cruise ship bans is beneficial to preventing the entry of people from other countries with the virus. The establishment of the temporary hospitals can improve the ability to accommodate quarantined patients. The release of prevention and control guidelines contributes to guiding people to take correct self-protection methods. Improving the production of medical supplies and the ability to test for COVID-19 ensures that patients can be detected and treated promptly, and increases recovery rates. Policy relaxation and economic restarts should be conducted after the epidemic situation is relatively stable, rather than prematurely. Large-scale activities with crowd gatherings should be cancelled as much as possible, owing to the existence of a large potential infection rate. At the resident level, some countries have promulgated corresponding measures, but citizens have not strictly implemented them. Some citizens resist the 'closing the city' measure, for they think it conflicts with personal freedom. Some people have negative emotions about the severe epidemic and determination of them to protest together is insufficient, affecting the policy response. It is essential to actively respond to the relevant prevention and control policies issued by the state, and not to violate the prohibition without authorisation. Although the current epidemic situation in some countries is gradually stable, keeping vigilant, paying attention to personal protection, and reducing long-distance travel remain important.

In the view of the actual situation and prediction, Chinese epidemic is in a stable state, whereas the United States is firmly in the first place worldwide; moreover, the dynamic trends of aggravation of the COVID-19 epidemic in India and Brazil are obvious. The total amount of confirmed cases and deaths in the United States accounted for approximately $21.95 \%$ and $18.40 \%$ of those worldwide on December 5, 2020, respectively; these values are estimated to change to $23.60 \%$ and $18.11 \%$, respectively, by January 16, 2021. Countries with severe epidemics such as the United States, Brazil, and India may suffer severe economic damage, market economic imbalances, factory or company closures, significantly increased unemployment, displacement of people, and increased hunger. To avoid gatherings of people, the elections in the United States will also be affected. In summary, the most effective measure is to self-protection, and the most basic protection is to wear a mask and keep a physical distance. In addition to effective prevention and control policies, only active self-protection can minimise the chance of infection. To prevent the global crisis caused by the continued deterioration of the epidemic, economic restarts, and resumptions of work and school should be avoided as much as possible before the epidemic has stabilised.

\section{Conclusions}

In this work, an LSTM-based neural network model was proposed to predict the dynamics of COVID-19 in China, five countries with severely epidemics, and the world. The error rate of our model was less than $1.4 \%$. We demonstrated that the $3 *$ LSTM model, with good fitting performance, is superior to other methods, and that the trend predictions basically conform 
to the actual current situations. According to forecasts, if the present measures are maintained, the amount of cumulative confirmed cases in the globe will exceed 84.58 million by January 16, 2021, with a global declining mortality rate.

Under the analysis of measures in China and the five other countries, untimely and inappropriate government policies, lax regulations, and insufficient public cooperation are the reasons for the aggravation of the epidemic situations. A summary is provided, as follows. First, it is vital for the government to take appropriate and proactive measures in time. Simultaneously, the active cooperation of the public, and faith in fighting the epidemic together is needed to promote the implementation of the measures for obtaining good effects. The first and most essential approach is to wear masks and keep a physical distance. Other active policies should be advocated, such as the release of emergency responses, lock-downs of cities and closures of entertainment venues, prohibitions of gatherings as much as possible, the establishment of temporary hospitals, border inspections, bans on cruise ships, prevention and control guidelines, the vigorously production of medical materials, and improvements in the ability to detect COVID-19. Second, the epidemic situations of the United States, India, and Brazil are not encouraging. Opening in advance, neglecting protective measures, having gatherings, and insufficient medical resources have provided great potential for infection. Third, in countries where the epidemic situation has not stabilised or is growing significantly, according to the stage of the epidemic, it is necessary to adjust policies flexibly, increase prevention and control efforts, expand the intensity of nucleic acid testing, deploy medical supplies, and avoid large-scale gatherings. In countries where the epidemic has gradually stabilised, to prevent the recurrence of the epidemic after the resumption of production, personal protection should be maintained, together with disinfection at the places of work, study and entertainment in time, along with body temperature monitoring of entry and exit personnel.

Only with the use of a vaccine will we be able to curb the spread and multiplication of the virus. However, the timing of emergence, effectiveness, and side effects of the vaccine are unknown. Thus, people must wear a mask and maintain a physical distance to take precautions against COVID-19 for the next two years, even with widespread vaccination.

\section{References}

1. Zhou, P. et al. A pneumonia outbreak associated with a new coronavirus of probable bat origin. Nature 579, $270-273$. https://doi.org/10.1038/s41586-020-2012-7 (2020).

2. The Secretary-General of the United Nations calls on everyone to unite: the new crown epidemic is the most serious global crisis facing humanity since World War II. https://world.chinadaily.com.cn/a/202004/01/WS5e83fa64a3107bb6b57aa057.html (accessed August 31, 2020).

3. World Health Organization. WHO Director-General's opening remarks at the media briefing on COVID-19 - 11 March 2020. https://www.who.int/zh/dg/speeches/detail/who-director-general-s-opening-remarks-at-the-media-briefing-on-covid-19---11-march-2020 (accessed August 13, 2020).

4. Gasmi, A. et al. Individual risk management strategy and potential therapeutic options for the COVID-19 pandemic. Clin. Immunol. 215, 108409. https://doi.org/10.1016/j.clim.2020.108409 (2020).

5. Wu, Y. Chen, C. \& Chan, Y. The outbreak of COVID-19: an overview. J. Chin. Med. Assoc. 83, 217-220 (2020).

6. Benvenuto, D. et al. The 2019 - new coronavirus epidemic: Evidence for virus evolution. J. Med. Virol. 92, $455-459$. https://doi.org/10.1002/jmv.25688 (2020).

7. Woods, JA. et al. The COVID-19 Pandemic and Physical Activity. Sport. Med. Health Sci. 2 , 55-64. https://doi.org/10.1016/j.smhs.2020.05.006 (2020).

8. Chen, N. et al. Epidemiological and clinical characteristics of 99 cases of 2019 novel coronavirus pneumonia in Wuhan, China: a descriptive study. Lancet 395, 507-513. https://doi.org/10.1016/S0140-6736(20)30211-7 (2020).

9. Juan, A \& Siordia, Jr. Epidemiology and clinical features of COVID-19: A review of current literature. J. Clin. Virol. 127, 104357. https://doi.org/10.1016/j.jcv.2020.104357 (2020).

10. Puntmann, V.O. et al. Outcomes of Cardiovascular Magnetic Resonance Imaging in Patients Recently Recovered From Coronavirus Disease 2019 (COVID-19). JAMA Cardiol. https://doi.org/10.1001/jamacardio.2020.3557 
(2020).

11. Mckibbin, W.J. \& Fernando, R. The Global Macroeconomic Impacts of COVID-19: Seven Scenarios. CAMA Working Paper No. 19/2020. http://dx.doi.org/10.2139/ssrn.3547729 (2020).

12. Abcnews. UN: Virus could push 14 million into hunger in Latin America. Available online: https://abcnews.go.com/Health/wireStory/virus-push-14-million-hunger-latin-america-70919402 (accessed August 13, 2020).

13. Qun, L. et al. Early Transmission Dynamics in Wuhan, China, of Novel Coronavirus-Infected Pneumonia. N. Engl. J. Med. 382, 1199-1207. https://doi.org/10.1056/NEJMoa2001316 (2020).

14. Yang, Z. et al. Modified SEIR and AI prediction of the epidemics trend of COVID-19 in China under public health interventions. J. Thorac. Dis. 12, 165-174. https://doi.org/10.21037/jtd.2020.02.64 (2020).

15. World Health Organization. Coronavirus disease (COVID-19) Weekly Epidemiological Update. https://www.who.int/publications/m/item/weekly-epidemiological-update-8-december-2020 (accessed December 9, 2020).

16. World Health Organization. Draft landscape of COVID-19 candidate vaccines. Available online: https://www.who.int/publications/m/item/draft-landscape-of-covid-19-candidate-vaccines (accessed December 9, 2020).

17. Li, Z. et al. Active case finding with case management: the key to tackling the COVID-19 pandemic. Lancet 396, 63-70. https://doi.org/10.1016/S0140-6736(20)31278-2 (2020).

18. $\mathrm{BBC}$ news. US election 2020: What is the presidential transition? Available online: https://www.bbc.com/news/electionus-2020-55045075 (accessed December 12, 2020).

19. Fang, Y. Nie Y. \& Penny, M. Transmission dynamics of the COVID - 19 outbreak and effectiveness of government interventions: A data - driven analysis. J. Med. Virol. 92, 645-659. https://doi.org/10.1002/jmv.25750 (2020).

20. Hu, Z. Ge, Q. Li, S. Jin, L. \& Xiong, M. Artificial Intelligence Forecasting of Covid-19 in China. Preprint at: https://arxiv.org/abs/2002.07112 (2020).

21. Goodfellow, I. Bengio, Y. \& Courville, A. Deep Learning. 367-415. http://www.deeplearningbook.org (2016).

22. Zandavi, S.M. Rashidi, T.H. \& Vafaee, F. Forecasting the Spread of Covid-19 Under Control Scenarios Using LSTM and Dynamic Behavioral Models. Preprint at: https://arxiv.org/abs/2005.12270 (2020).

23. National Health Commission of the people's Republic of China. Report of the epidemic situation (in Chinese). Available online: http://www.nhc.gov.cn/xcs/yqtb/list_gzbd.shtml (accessed August 31, 2020).

24. Health Commission of Hubei Province. The epidemic situation of new crown pneumonia in Hubei Province (in Chinese). Available online: http://wjw.hubei.gov.cn/bmdt/dtyw/ (accessed August 31, 2020).

25. Johns Hopkins University in the United States. Open data sets. Available online: https://github.com/CSSGISandData/COVID-19 (accessed August 31, 2020).

26. Baidu. Real-time big data report on the epidemic (in Chinese). Available online: https://voice.baidu.com/act/newpneumonia/newpneumonia/?from=osari_pc_3 (accessed August 31, 2020).

27. Hochreiter, S \& Schmidhuber, J. Long Short-Term Memory. Neural Computation 9, 1735-1780. https://doi.org/10.1162/neco.1997.9.8.1735 (1997).

28. Olah, C. Understanding LSTM Networks. Blog Network. Available online: http://colah.github.io/posts/2015-08Understanding-LSTMs/ (accessed August 14, 2020).

29. Wan, K. et al. When will the battle against novel coronavirus end in Wuhan: A SEIR modeling analysis. J. Glob. Health 10. https://doi.org/10.1101/2020.02.16.20023804 (2020).

30. Huang, C. et al. Clinical features of patients infected with 2019 novel coronavirus in Wuhan, China. Lancet 395, 497-506. https://doi.org/10.1016/S0140-6736(20)30183-5 (2020).

31. Chinese Center for Disease Control and Prevention. Guidelines for COVID-19 epidemiological investigations. http://weekly.chinacdc.cn/fileCCDCW/journal/article/ccdcw/2020/19/PDF/Annex-1.pdf (accessed August 14, 2020).

32. Chinese Center for Disease Control and Prevention. COVID-19 Prevention and Control Guide (First Edition) (in Chinese). Available online: http://www.chinacdc.cn/jkzt/crb/zl/szkb_11803/jszl_2275/202002/t20200201_212138.html (accessed 
August 14, 2020).

33. The State Council Information Office of the People's Republic of China. Full Text: Fighting COVID-19: China in Action. Available online: http://www.scio.gov.cn/zfbps/32832/Document/1681809/1681809.htm (accessed October 17, 2020)

34. Holshue, M.L. et al. First Case of 2019 Novel Coronavirus in the United States. N. Engl. J. Med. 382, 929-936. https://doi.org/10.1056/NEJMoa2001191 (2020).

35. National Immigration Administration. Remind on recent immigration control measures of relevant countries (in Chinese). Available online: https:/www.nia.gov.cn/n794014/n1050181/n1050489/c1214826/content.html (accessed August 14, 2020).

36. Anne Schuchat, MD \& CDC COVID-19 Response Team. Public Health Response to the Initiation and Spread of Pandemic COVID-19 in the United States, February 24 - April 21, 2020. J. MMWR. Morbidity and Mortality Weekly Report. 69(18). https://www.cdc.gov/mmwr/volumes/69/wr/mm6918e2.htm (2020).

37. Centers for Disease Control and Prevention. Coronavirus disease 2019 (COVID-19). Recommendation for cloth face covers. Atlanta, GA: US Department of Health and Human Services. https://www.cdc.gov/coronavirus/2019ncov/prevent-getting-sick/cloth-face-cover.html (2020).

38. Goodmorningamerica. Hospitalizations rise as Trump pushes reopening. 2020. Available online: https:/www.goodmorningamerica.com/news/video/hospitalizations-rise-trump-pushes-reopening-72048700 (accessed August 14, 2020).

39. Human Resources and Social security Department of Hubei Province. The decision on awarding Dingyu Zhang and Jixian Zhang for great achievements (in Chinese). Available online: http://m.hbdysh.cn/article/25927 (accessed August 14, 2020).

40. HUBEI.GOV.CN. Announcement of Wuhan City's COVID-19 Epidemic Prevention and Control Headquarters (in Chinese). Available online: http://www.hubei.gov.cn/zhuanti/2020/gzxxgzbd/zxtb/202001/t20200123_2014402.shtml (accessed August 14, 2020).

41. People.cn. Provide strong legal protection for epidemic prevention and control (in Chinese). http://yuqing.people.com.cn/n1/2020/0211/c429781-31580633.html (accessed August 14, 2020).

42. Chinanews. From "Huoshenshan", "Leishenshan" to the "Fangcang" hospital, race against the epidemic at the speed of China! (in Chinese). Available online: http://www.chinanews.com/sh/shipin/cns/2020/02-05/news846922.shtml (accessed August 14, 2020).

43. HUBEI.GOV.CN. At 0:00 today, Wuhan "unblocked" (in Chinese). Available online: http://www.hubei.gov.cn/2019/tpyW/202004/t20200408_2207205.shtml (accessed August 14, 2020).

44. CCTV.com. The National Health Commission reminds again during the May Day holiday: time-sharing appointments for peak-shift trips. Available online: http://news.cctv.com/2020/05/02/ARTIlsOonxEyXZQdSG4ioeV6200502.shtml (accessed August 14, 2020).

45. Jernigan DB \& CDC COVID-19 Response Team. Update: public health response to the coronavirus disease 2019 outbreak-United States, February 24, 2020. Morbidity and Mortality Weekly Report. 69:216-9. https://doi.org/10.15585/m-mwr.mm6908e1 (accessed August 14, 2020).

46. Abcnews. Mass protests could lead to another wave of coronavirus infections. Available online: https://abcnews.go.com/US/mass-protests-lead-wave-coronavirus-infections/story?id=70997184 (accessed August 14, 2020).

47. Goodmorningamerica. Trump reacts after low turnout at Tulsa rally. Available online: https:/www.goodmorningamerica.com/news/video/trump-reacts-low-turnout-tulsa-rally-71379690 (accessed August 14, 2020).

48. Xinhuanet. Spotlight: More U.S. states mandate wearing masks in public as COVID-19 cases continue to rise. Available online: http://www.xinhuanet.com/english/2020-06/25/c_139166307.htm (accessed October 14, 2020).

49. Xinhuanet. "Restart" epidemic briefing. Trump calls for wearing masks (in Chinese). Available online: http://m.xinhuanet.com/world/2020-07/23/c_1210716033.htm (accessed October 14, 2020). 
50. Only one week after school starts, the number of confirmed cases in U.S. universities surges. Available online: http://www.xinhuanet.com/video/2020-08/26/c_1210770179.htm (accessed August 31, 2020).

51. Goodmorningamerica. Here's everyone who's tested positive for COVID-19 in connection to the White House. Available online: https://www.goodmorningamerica.com/news/video/heres-tested-positive-covid-19-connection-white-house-73512771 (accessed October 14, 2020).

52. Theguardian. Health secretary: 'Window is closing' to stop coronavirus as US cases pass 2.5m. 2020. Available online: https://www.theguardian.com/world/2020/jun/28/us-health-secretary-window-closing-stop-coronavirus-cases-pass-25m (accessed August 14, 2020).

53. The paper. Brazil's epidemic timeline: President takes the lead in ignoring epidemic prevention measures and finally infects the COVID-19 (in Chinese). Available online: https://www.thepaper.cn/newsDetail_forward_8174050 (accessed August 14, 2020).

54. Xinhuanet. The first professional football match at Rio, Brazil after the epidemic (in Chinese). Available online: http://sports.xinhuanet.com/c/2020-06/19/c_1126136210.htm (accessed August 14, 2020).

55. Xinhuanet. Epidemic rebounds, "Silicon Valley of India" and other places are "closed" again (in Chinese). Available online: http://www.xinhuanet.com/world/2020-07/16/c_1210703949.htm (accessed August 14, 2020).

56. Abcnews. The Latest: India surpasses 150,000 virus cases. Available online: https://abcnews.go.com/Health/wireStory/latest-india-reports-biggest-jump-virus-cases-70876706 (accessed August 14, 2020).

57. Kankanews. The number of confirmed cases soared to 20,000. Why did the Russian epidemic suddenly worsen? (in Chinese). Available online: http://www.kankanews.com/a/2020-04-14/0039217322.shtml (accessed August 14, 2020).

58. Xinhuanet. Hot Q\&A: Two highlights of the Russian military parade on Victory Day (in Chinese). Available online: http://www.xinhuanet.com/world/2020-06/24/c_1126154264.htm (accessed August 14, 2020).

59. Gouvernement.fr. The Government's actions. https://www.gouvernement.fr/info-coronavirus/les-actions-dugouvernement (accessed November 7, 2020).

60. CNN. ICUs are nearing capacity in this French city. And it's only September. https:/edition.cnn.com/2020/09/16/europe/france-bordeaux-coronavirus-hospitals-intl/index.html (accessed November 7 , 2020).

\section{Acknowledgements}

This work was supported in part by the National Key R\&D Program of China under Grant 2017YFC150090501, the National Natural Science Foundation of China under Grant 41871325.

\section{Author contributions}

Conceived and designed the experiments: Z.H, Z.F. Performed the experiments: Z.H, Z.F. R.Z. Supervision of the experiments: X.T., Analyzed the data: Z.H, Z.F. R.Z. Wrote the paper: Z.H, Z.F., with the help of rest of authors.

\section{competing interests}

The authors declare no competing interests. 


\section{Figures}

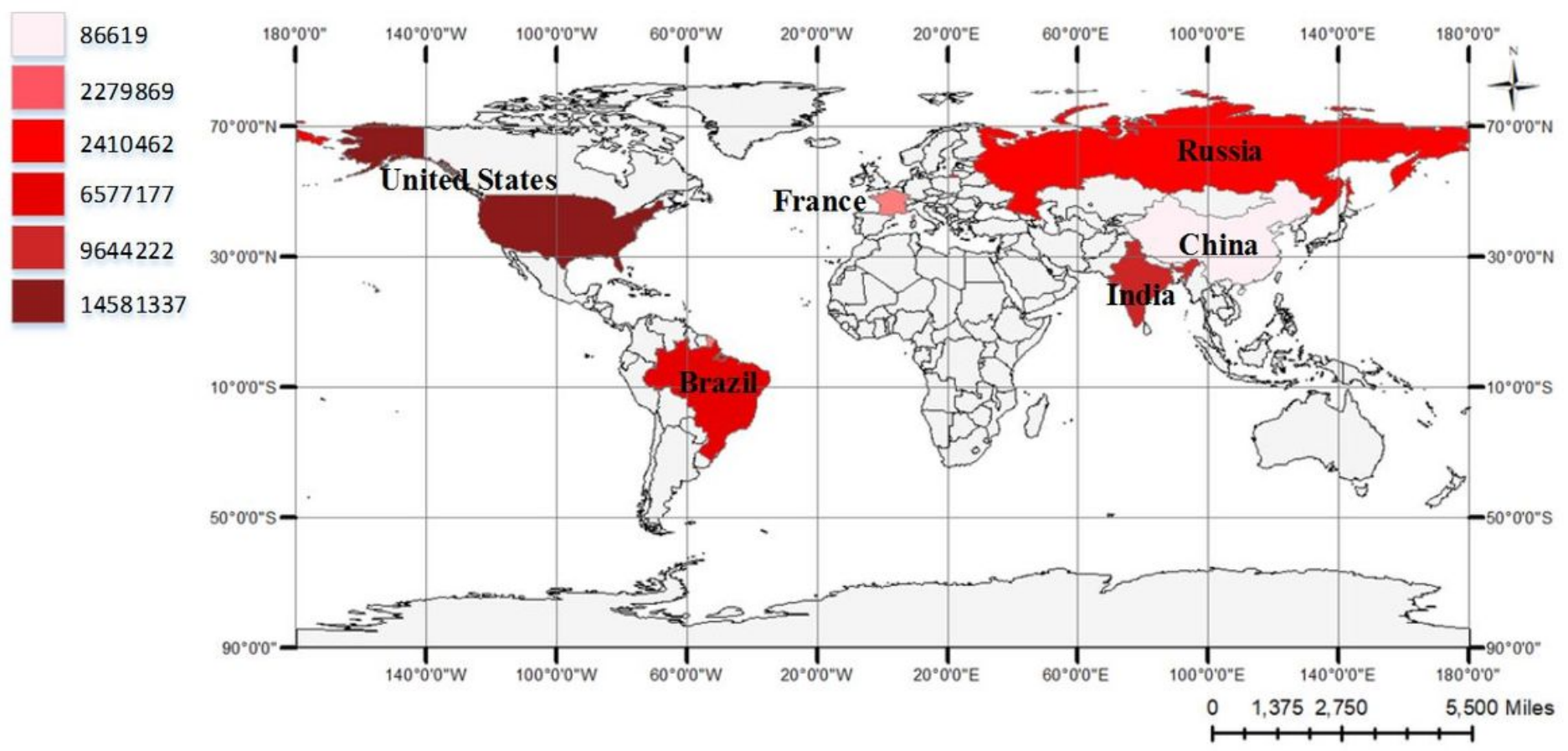

\section{Figure 1}

China and top five countries of confirmed COVID-19 cases worldwide. The data on the left represents the amount of cumulative confirmed cases in China and five countries with severe epidemic situation as of December 5, 2020. COVID-19, coronavirus disease 2019. Note: The designations employed and the presentation of the material on this map do not imply the expression of any opinion whatsoever on the part of Research Square concerning the legal status of any country, territory, city or area or of its authorities, or concerning the delimitation of its frontiers or boundaries. This map has been provided by the authors. 


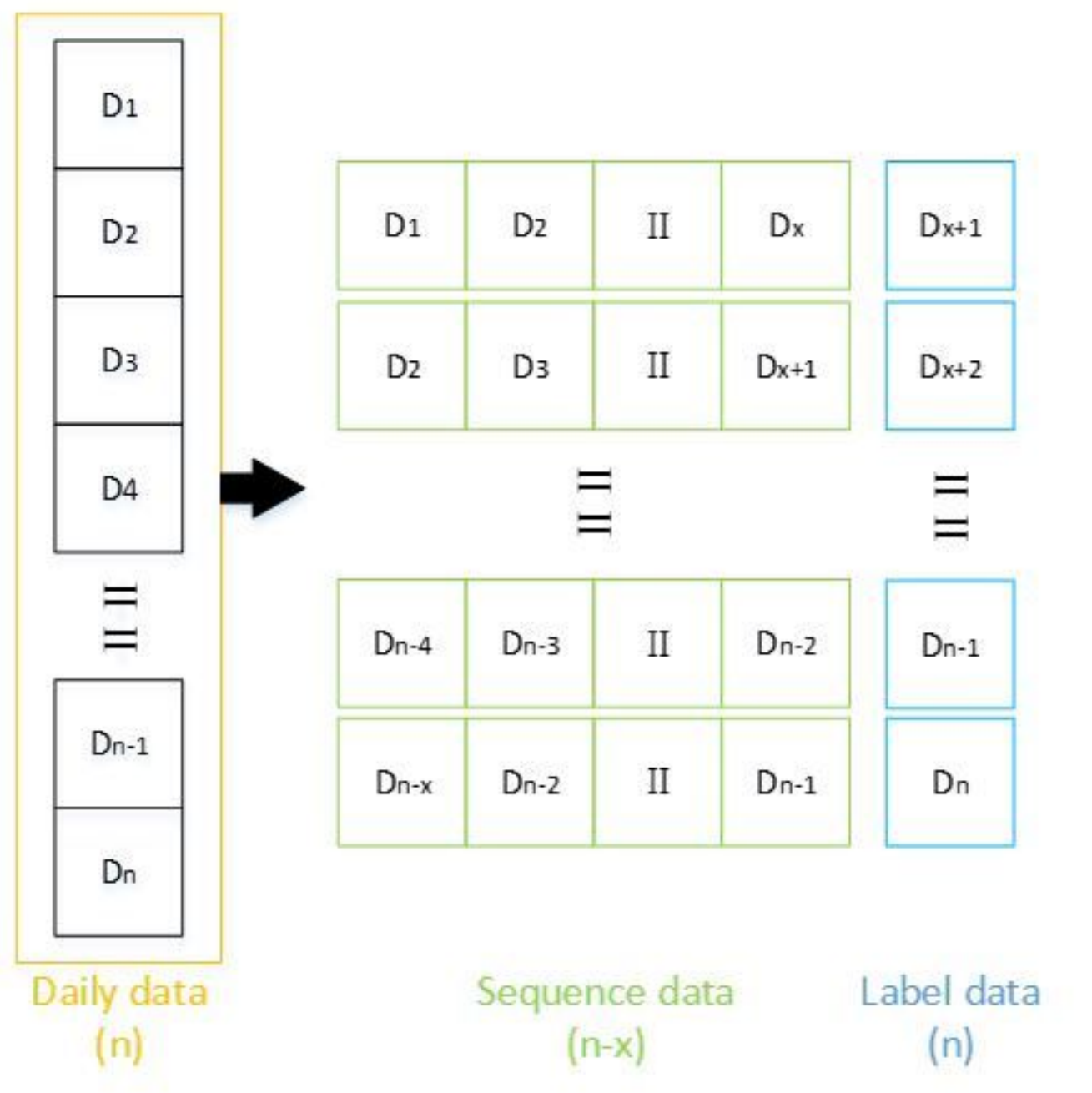

Figure 2

Sequence Data Generation

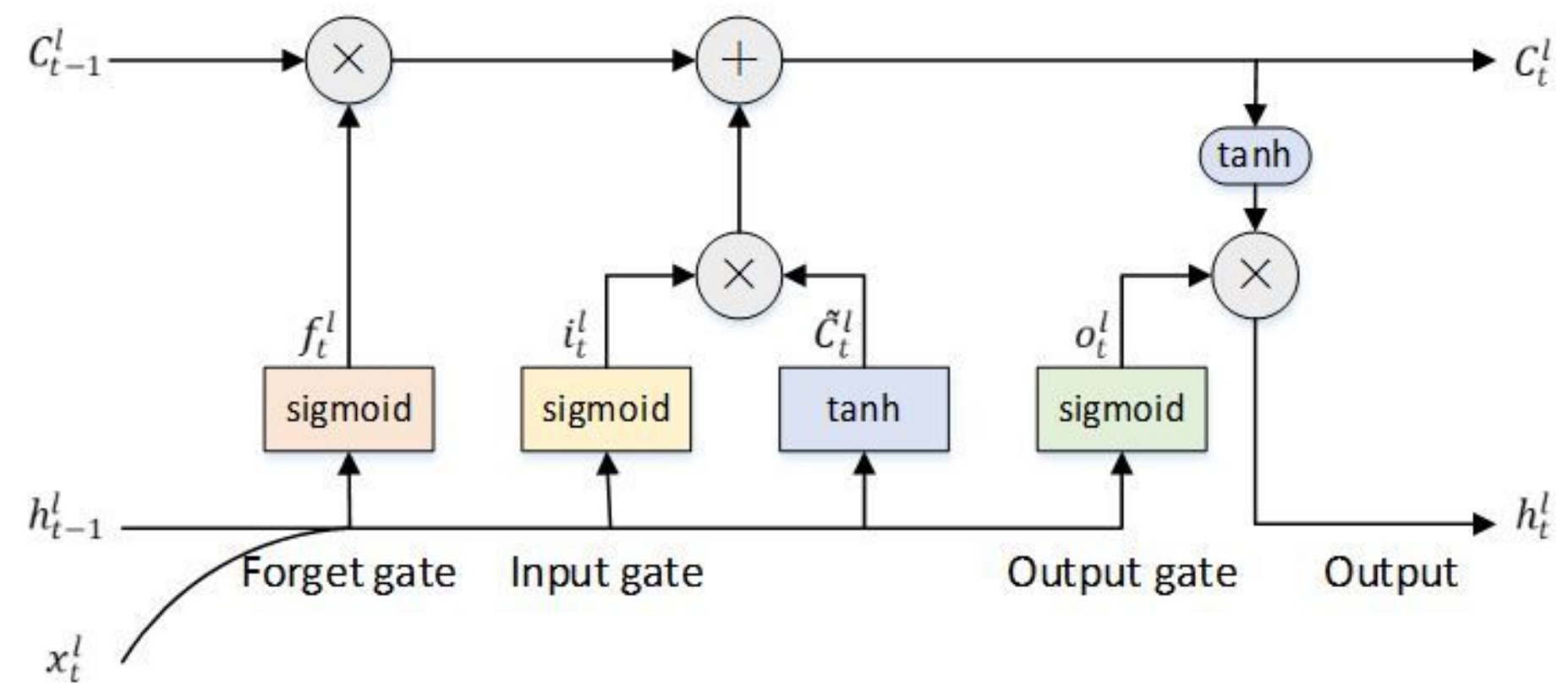


Figure 3

The inner structure of LSTM. LSTM, Long-Short-Term-Memory.
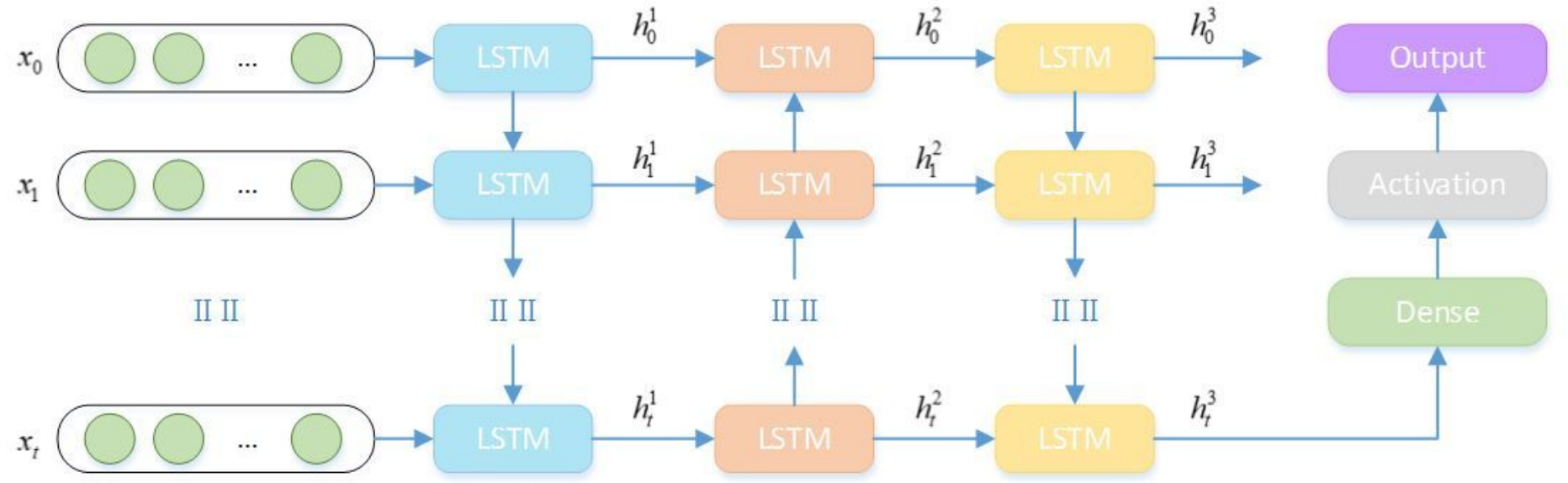

II II

Dense

Figure 4

LSTM model of recurrent expansion. $x$ indicates the sequence data, $h$ represents the output of the LSTM recurrent unit. The blue is the first LSTM layer, orange is the second LSTM layer, and yellow is the third LSTM layer. LSTM, Long-Short-Term-Memory.

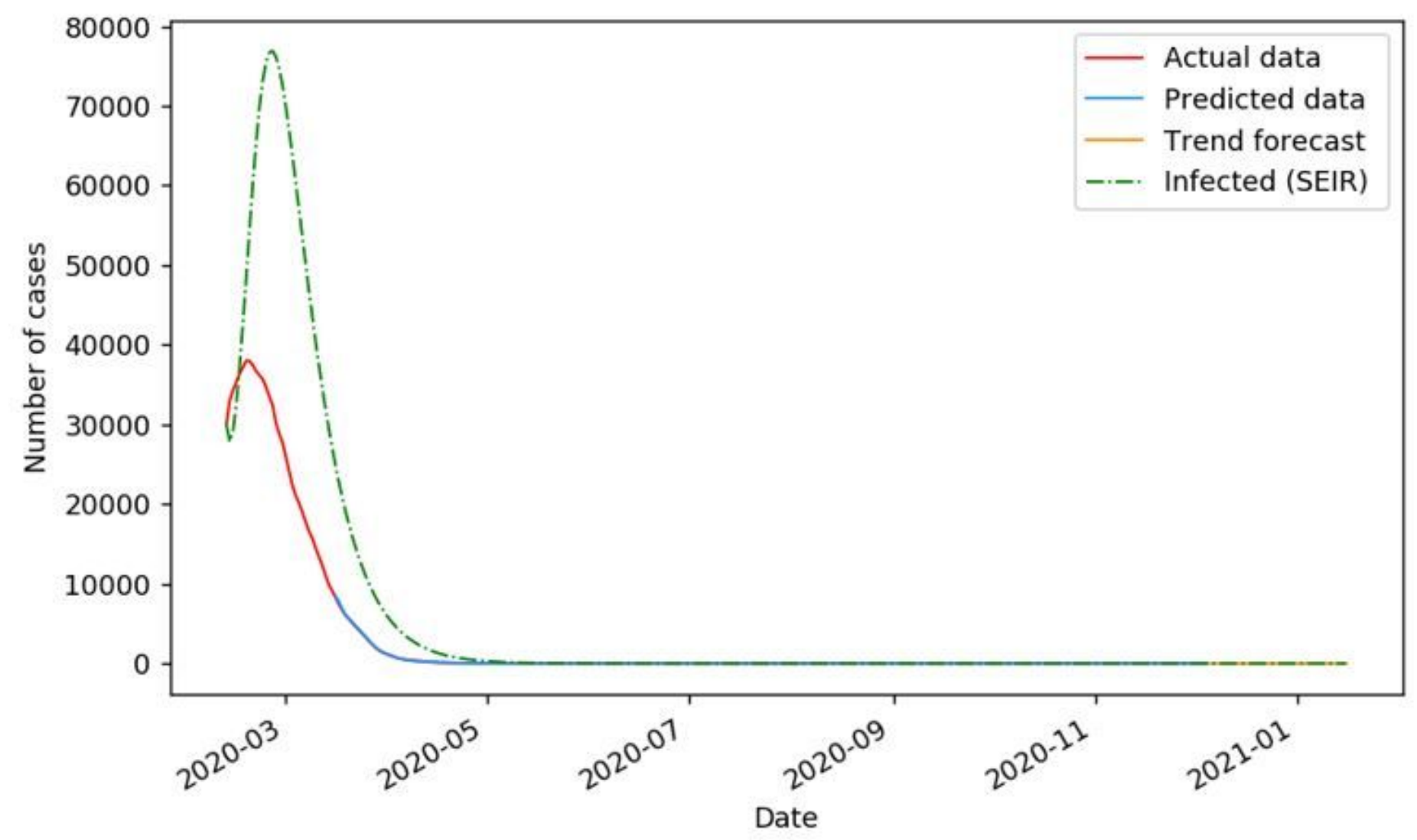

Figure 5 
Comparison of $3^{\star}$ LSTM model and SEIR model19 in the current confirmed COVID-19 cases of Wuhan, Hubei Province, China. The solid red line is real data, the solid blue line represents the predicted data with label data training in the LSTM model, the solid green line indicates the trend prediction in the LSTM model, and the dashed orange line denotes the number of infected individuals as predicted by the SEIR model. LSTM, Long-Short-Term-Memory; SEIR, Susceptible-Exposed-Infectious-Removed. COVID-19, coronavirus disease 2019.

a

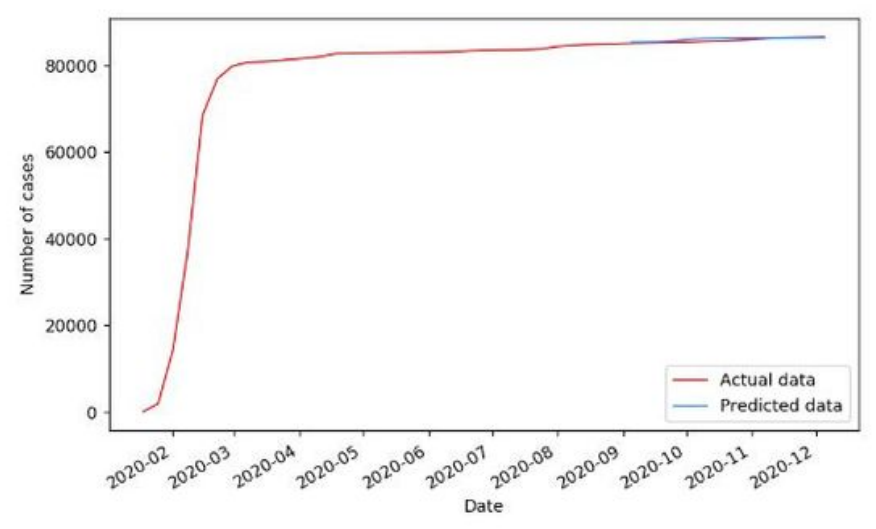

b

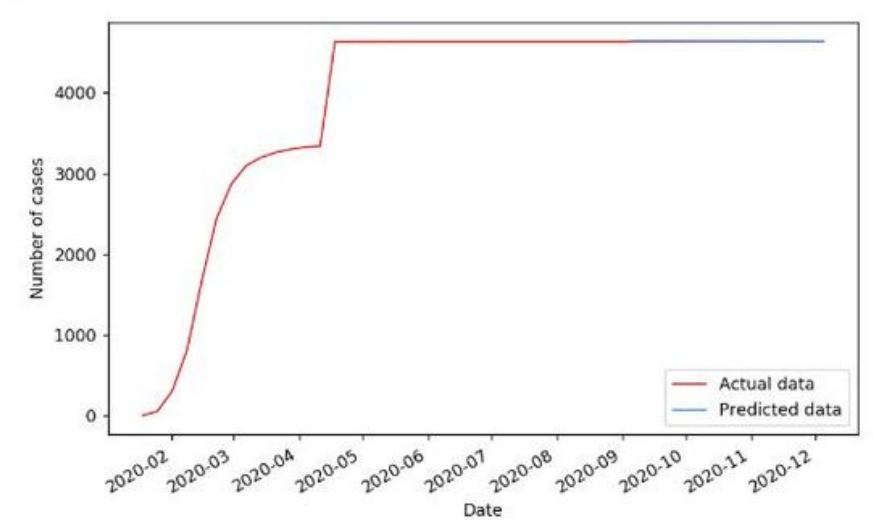

Figure 6

Prediction of cumulative COVID-19 cases in China and the United States by $3 *$ LSTM model. (a) Cumulative confirmed cases in China. (b) Cumulative deaths in China. LSTM, Long-Short-Term-Memory. COVID-19, coronavirus disease 2019.

a

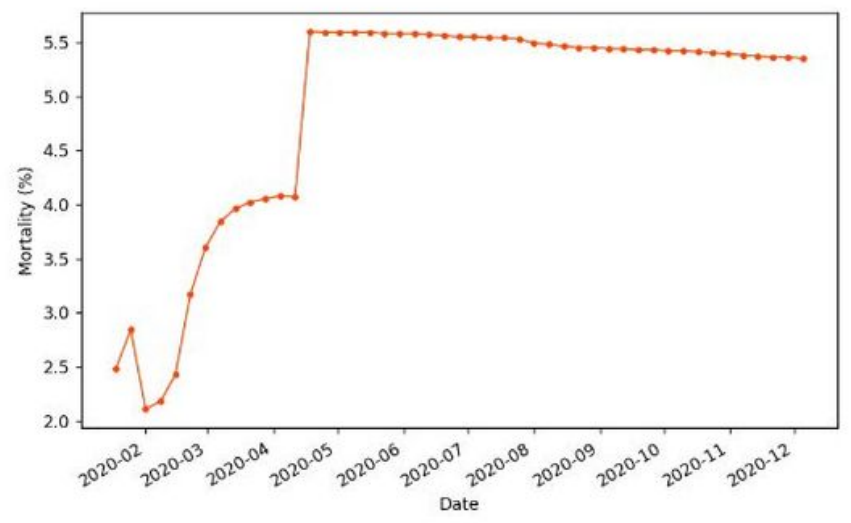

b

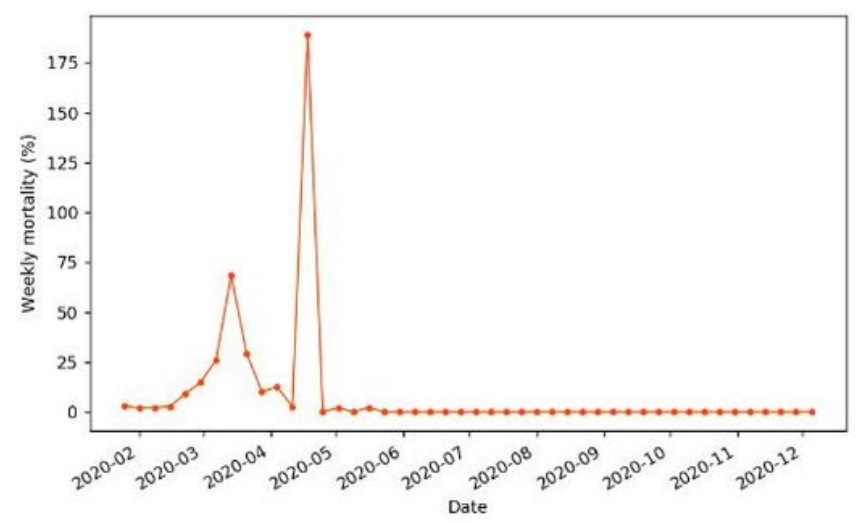

Figure 7

The mortality of COVID-19 cases in China and the United States. (a) Cumulative mortality. (b) Weekly mortality: the rate of weekly new deaths and new cases. COVID-19, coronavirus disease 2019. 
a

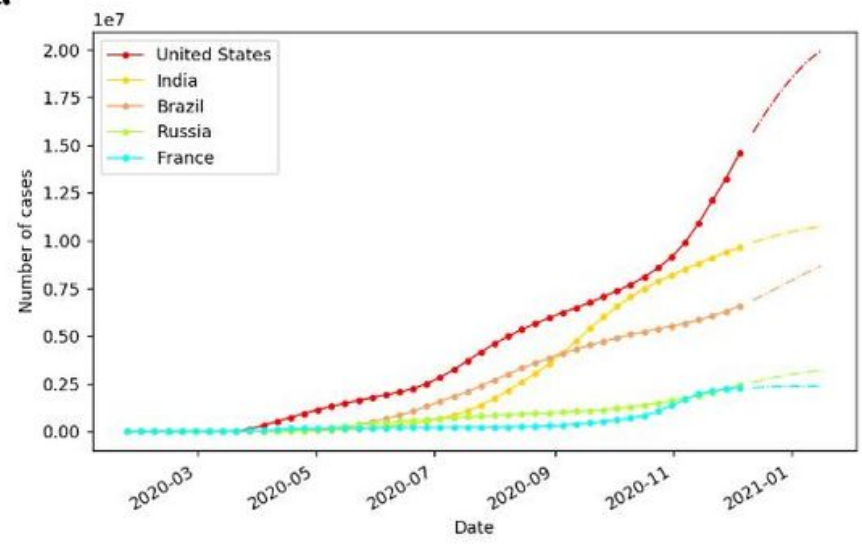

b

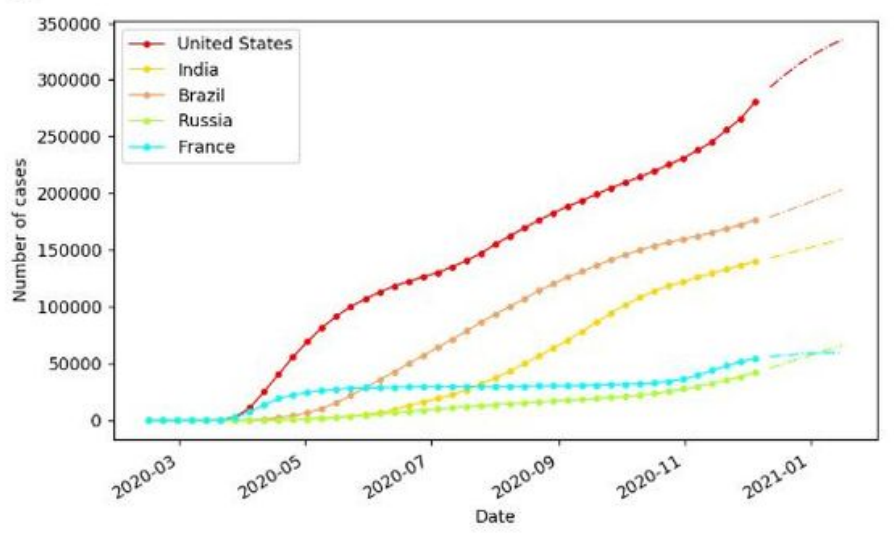

\section{Figure 8}

Prediction of cumulative COVID-19 cases in five countries by 3*LSTM model. (a) Prediction of weekly cumulative confirmed cases. (b) Prediction of weekly cumulative death cases. The solid line is the actual data, and the dotted line is the forecast data. LSTM, Long-Short-Term-Memory; COVID-19, coronavirus disease 2019.

a

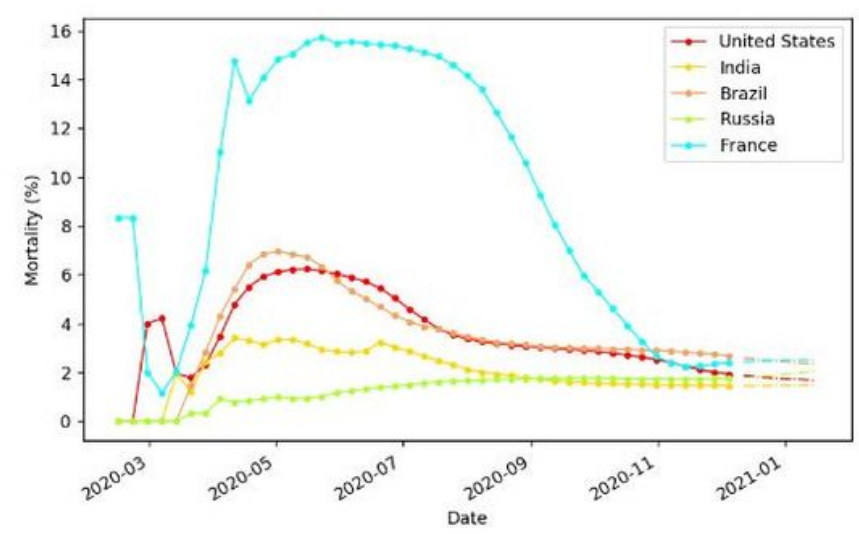

b

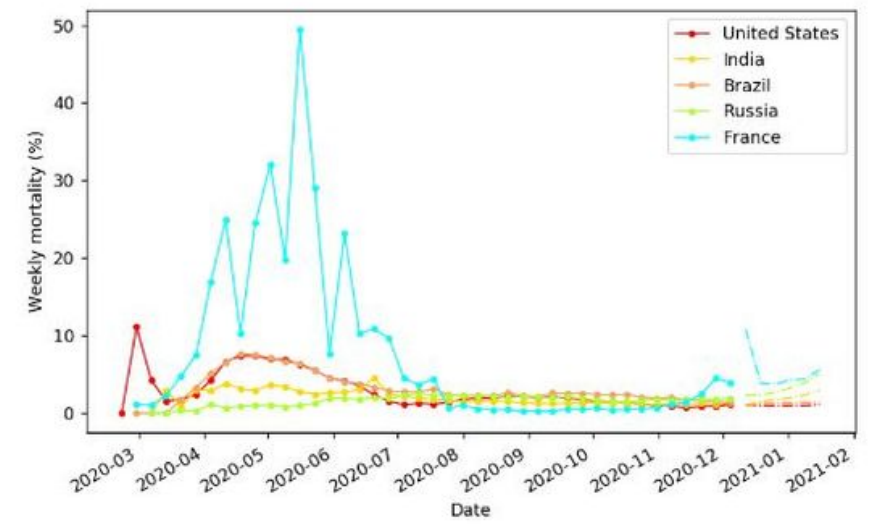

Figure 9

Mortality comparison of COVID-19 patients in five countries. (a) Cumulative mortality. (b) Weekly mortality: the rate of weekly new deaths and new cases. The solid line represents the actual mortality, and the dotted line represents the predicted mortality by $3 *$ LSTM model. COVID-19, coronavirus disease 2019; LSTM, Long-Short-Term-Memory. 


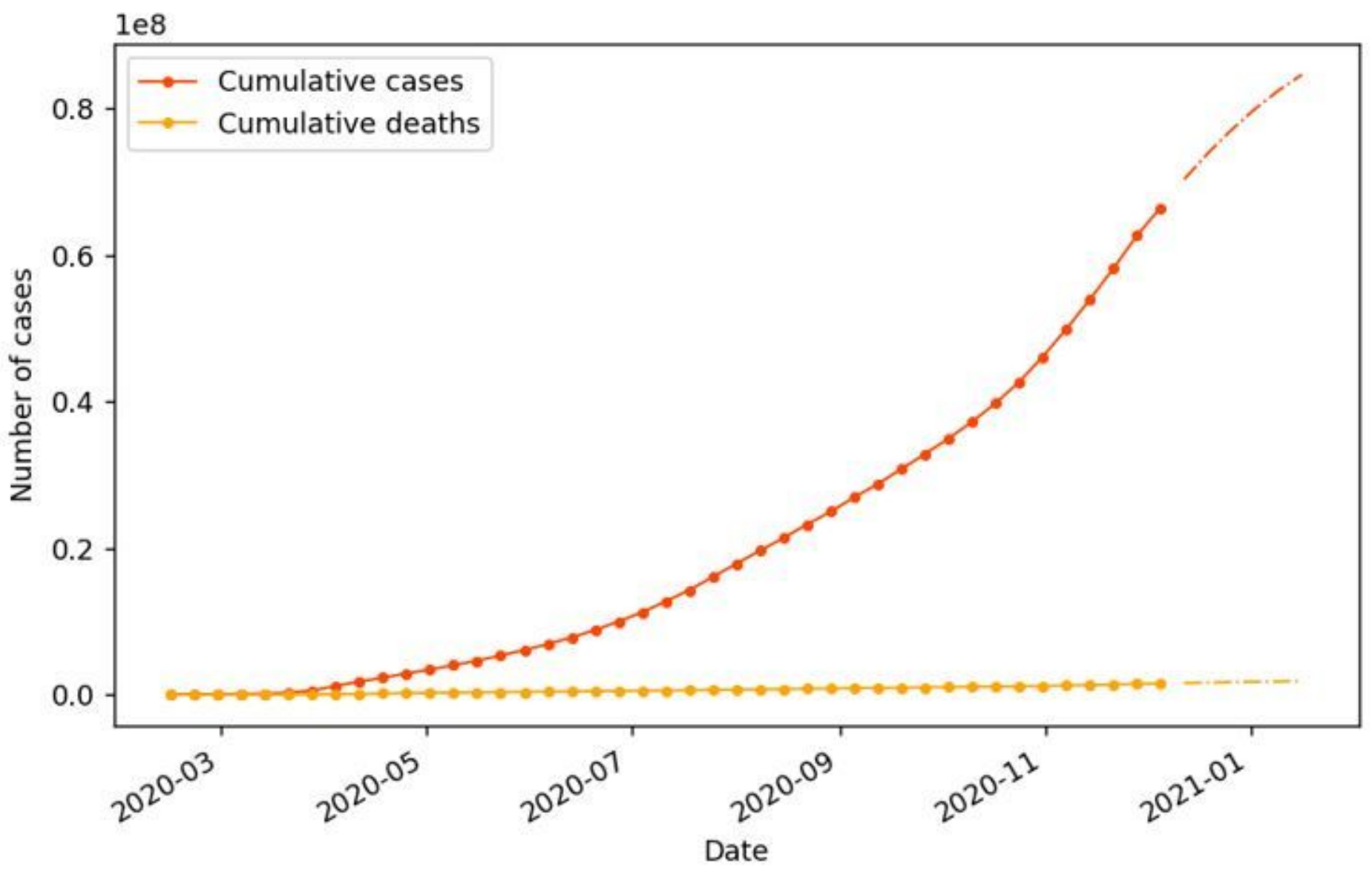

Figure 10

Prediction of global cumulative COVID-19 cases by $3 *$ LSTM model. The solid line is actual data, the dotted line represents the predicted data. LSTM, Long-Short-Term-Memory; COVID-19, coronavirus disease 2019.

a

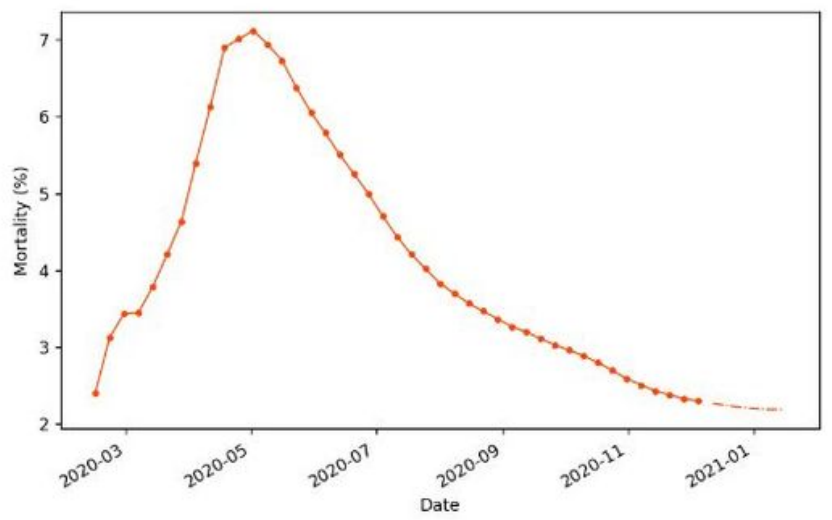

b

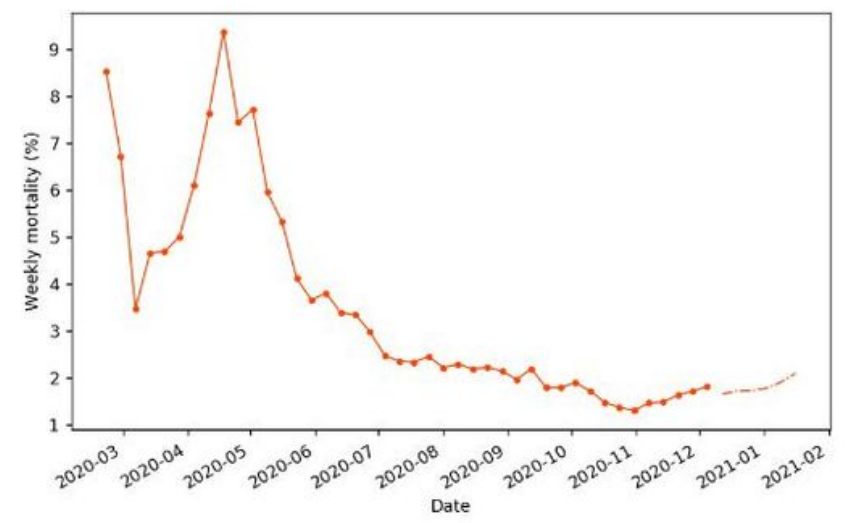

\section{Figure 11}

Forecast of global COVID-19 mortality. (a) Cumulative mortality. (b) Weekly mortality: the rate of weekly new deaths and new cases. The solid line is actual data, the dotted line represents the predicted data. 
COVID-19, coronavirus disease 2019. 